\title{
Flow Separation Control Over a Ramp Using Sweeping Jet Actuators
}

\author{
Mehti Koklu* and Lewis R. Owens $^{\dagger}$ \\ NASA Langley Research Center, Hampton, VA 23681
}

\begin{abstract}
Flow separation control on an adverse-pressure-gradient ramp model was investigated using various flow-control methods in the NASA Langley 15-Inch Wind Tunnel. The primary flow-control method studied used a sweeping jet actuator system to compare with more classic flow-control techniques such as micro-vortex generators, steady blowing, and steady- and unsteady-vortex generating jets. Surface pressure measurements and a new oilflow visualization technique were used to characterize the effects of these flow-control actuators. The sweeping jet actuators were run in three different modes to produce steadystraight, steady-angled, and unsteady-oscillating jets. It was observed that all of these flowcontrol methods are effective in controlling the separated flows on the ramp model. The steady-straight jet energizes the boundary layer by momentum addition and was found to be the least effective method for a fixed momentum coefficient. The steady-angled jets achieved better performance than the steady-straight jets because they generate streamwise vortices that energize the boundary layer by mixing high-momentum fluid with near wall lowmomentum fluid. The unsteady-oscillating jets achieved the best performance by increasing the pressure recovery and reducing the downstream flow separation. Surface flow visualizations indicated that two out-of-phase counter-rotating vortices are generated per sweeping jet actuator, while one vortex is generated per vortex-generating jets. The extra vortex resulted in increased coverage, more pressure recovery, and reduced flow separation.
\end{abstract}

$\begin{array}{lll}A R & = & \text { actuator orifice aspect ratio } \\ A_{\text {jet }} & = & \text { area of total jet orifices } \\ A_{\text {sep }} & = & \text { area of the separated region } \\ C m & = & \text { momentum coefficient } \\ C p & = & \text { pressure coefficient } \\ C R J & = & \text { circular round jet } \\ \Delta C p / \Delta x & = & \text { pressure gradient parameter } \\ F R & = & \text { reduced frequency of pulsed jet } \\ M V G & = & \text { micro-vortex generator } \\ Q & = & \text { volumetric flow rate } \\ S W J & = & \text { sweeping jet } \\ S T J & = & \text { steady straight jet } \\ U_{\text {jet }} & = & \text { jet velocity out of actuators } \\ V G J & = & \text { vortex-generating jet } \\ V R & = & \text { jet-to-tunnel velocity ratio } \\ V_{\text {onset }} & = & \text { onset flow velocity } \\ V_{\text {tunnel }} & = & \text { tunnel flow velocity } \\ " & = & \text { inch }\end{array}$

Nomenclature

I. Introduction

Boundary-layer flow control has the potential to improve the performance (through lift enhancement, drag reduction, transition delay, separation delay, mixing enhancement, heat-transfer enhancement, and noise

\footnotetext{
* Research Engineer, Flow Physics and Control Branch, MS 170, Member AIAA.

${ }^{\dagger}$ Research Engineer, Flow Physics and Control Branch, MS 170, Senior Member AIAA.
}

1

American Institute of Aeronautics and Astronautics 
suppression) of many engineering systems (wings, fan blades, ducts, etc.). Typically, a boundary layer separates when the fluid in the boundary layer does not have enough momentum to overcome the kinetic energy losses associated with the adverse-pressure gradient and the viscous dissipation of the particular application. Due to the large energy losses associated with the boundary-layer separation, the performance of a system may be greatly reduced in the form of increased drag, reduced lift, or reduced total pressure recovery. Separation control methods are usually designed to energize the decelerated near-wall fluid within the boundary layer. The primary methods of energizing the boundary layer, and hence controlling the flow separation are the addition of momentum and vorticity to the boundary layer. Adding linear momentum to the decelerated fluid directly increases near-wall fluid momentum and thus delays or completely eliminates the flow separation. Near-wall momentum addition in the form of blowing has been a preferred and straightforward separation-control technique. It has been extensively studied in the literature. ${ }^{1}$ Adding streamwise vorticity increases the boundary layer mixing and therefore enhances the convective transport of the free stream momentum towards the near wall. Vorticity addition can be either passive or active. Passive micro-vortex generators (MVGs) have been successfully utilized ${ }^{2}$ in both low-speed ${ }^{3-4}$ and highspeed $^{5}$ applications to control the boundary-layer flow. Lin performed a detailed review of vortex generator studies to control the boundary-layer separation. ${ }^{6}$ Active vortex-generating methods such as vortex-generating jets (VGJs) ${ }^{7}$ provide the benefit of the vortex-generators without having the detrimental parasitic drag as well as the opportunity to adjust the flow control parameters to maintain effectiveness with changing flow conditions. In addition to VGJs, other unsteady actuation methods such as oscillatory-blowing valves, ${ }^{8}$ pulsed VGJs, ${ }^{9-11}$ plasma actuators, ${ }^{12-14}$ synthetic jets, ${ }^{15}$ and fluidic oscillators ${ }^{16}$ introduce vortices and/or linear momentum to the boundary layer to achieve enhanced system performance by controlling the boundary layer with potentially lower mass-flow requirements than the steady blowing methods.

Fluidic oscillators, or sweeping-jet (SWJ) actuators, were primarily developed as a fluid amplifier, but are found in many diverse applications such as windshield-washer fluid nozzles, ${ }^{17}$ flow-metering devices, ${ }^{18-19}$ shower heads, and hot tubs. Although they have no moving parts, output from these actuators is an unsteady oscillating flow field. The SWJ actuators are becoming more popular in the active flow-control field due to their inherent design features including a simple structure, maintenance free, dimensional scalability, high frequency, and amplitude bandwidths. Recent numerical and experimental studies ${ }^{20-23}$ showed interesting features of this actuator. Performance improvements of about $60 \%$ have resulted from successful flow-control applications of the SWJ actuators as demonstrated on a single-element high-lift airfoil, ${ }^{24}$ on a V-22 wing-nacelle combination, ${ }^{25}$ and on wind turbine blades. ${ }^{26}$ They have been successfully used to reduce drag by $20 \%$ on trucks ${ }^{27}$ and by $16 \%$ on a bluff body. ${ }^{28}$ The SWJ actuators were applied on a vertical tail model (NACA 0012 airfoil section) of a typical twin-engine aircraft at Reynolds number up to 1.5 million and a $50-70 \%$ increase in the side force was reported. ${ }^{29}$

The purpose of the present study is to investigate how well the SWJ actuator functions as a flow-separation control device. In addition, the effectiveness of the SWJ actuators will be evaluated and compared with other flowcontrol devices (MVGs, steady blowing, steady and unsteady VGJs) applied to an adverse-pressure-gradient ramp flow.

\section{Experimental Setup and Methods}

The experimental data in this study consists of two different data sets acquired at two different time periods. The first data set was conducted by the second author in support of the NASA Ultra-Efficient Engine Technology (UEET) program in 2002. The first data set includes studies of flow-separation control using MVGs, steady and unsteady blowing. The second data set was conducted by the first author in support of the NASA Fixed-Wing Project in 2013. The second data set includes studies of flow-separation control using the SWJ actuators in steady and unsteady modes of operation. The same tunnel configurations (ramp, splitter plates, flap angle, tunnel speed, etc.) were used in both experimental studies. The baseline (no flow-control) configurations have been tested many times to ensure that the uncontrolled pressure distributions match with each other.

\section{A. Facility and Model}

Both experiments (2002 and 2013) were conducted in the NASA Langley 15-Inch Low-Speed Tunnel. As described in Ref. 4, this tunnel is a closed-return, atmospheric facility used primarily for fundamental flow physics research. The tunnel flow speed $\left(\mathrm{V}_{\text {tunnel }}\right)$ entering the test section is approximately $100 \mathrm{feet} / \mathrm{second}$. The ramp model was placed between $\mathrm{x}=58$ " to 75 " stations (see Fig. 1) and designed to have adverse pressure gradient with separated flow. The upstream splitter plate was raised 3.5" to accommodate the ramp model. The upstream boundary layer on the splitter plate develops before being subjected to the adverse pressure gradient. The flow above the splitter plate 
accelerated to provide an onset flow velocity ( $\mathrm{V}_{\text {onset }}$ ) of 140 feet/second just ahead of the ramp. The pressure distribution over the upper splitter plate was adjusted to be uniform between $x=20$ " to 50 " stations by using supports in the upper tunnel wall to slightly modify the ceiling geometry.

\section{B. Diagnostic Tools}

Static pressure ports $(0.020 "$ ID) were instrumented on the ramp model to provide pressure data. These pressure ports were located along the centerline of the ramp. There were four additional pressure ports laterally across the ramp at selected stations that were equally spaced at 0.5 " intervals around the centerline. The pressure ports were connected to a electronically-scanned-pressure (ESP) module that communicates with a computer via Ethernet. The pressure transducer in the acquisition module can record a maximum of 1 psid with an accuracy of \pm 0.06 psi. Surface pressure data presented in this study was obtained by ensemble averaging of 6400 pressure signals from each pressure port to provide excellent accuracy and filtering.

A new surface oil-flow visualization technique was developed and used in the 2013 study. The surface oil-flow visualization was obtained using a mixture of aviation oil, kerosene, and nano-sized fumed silica particles. The aviation oil serves as the primary agent similar to a typical fluorescent oil-film technique. It has fluorescent pigment that glows under the ultra-violet (UV) light and acts as a tracing media. Kerosene is used as a carrier fluid. It mixes very well with aviation oil (miscible) and modifies the oil viscosity. When the kerosene is evaporated, it leaves oil traces behind. Here the nano-silica particles should not be confused with the titanium dioxide $\left(\mathrm{TiO}_{2}\right)$ particles in a well-known kerosene $+\mathrm{TiO}_{2}$ flow visualization technique. While $\mathrm{TiO}_{2}$ particles serve as powdered pigment, the silica particles in this method serve as an anti-sagging agent, which is commonly used in household paints and coatings. As an anti-sagging agent, the silica particles keep the oil on the ramp surface after the tunnel is turned off. Therefore the oil does not flow under the influence of gravity and hence eliminates in-situ photographing requirements. The mixture can be applied on contact paper and be photographed later to eliminate any camera parallax, accessibility, and focusing problems. Since the tracing media is oil, similar to the fluorescent oil-film technique, the mixture does not dry out quickly and the flow visualization does not have the hysteresis effect as we usually see in the kerosene $+\mathrm{TiO}_{2}$ visualization technique. Using this mixture, the flow patterns are visible under both white and UV lights. When illuminated with the UV light, it provides brilliant surface flow patterns, whereas the model and background remain invisible. It has the advantages of kerosene $+\mathrm{TiO}_{2}$ and fluorescent oil-film techniques.

The new mixture was applied on contact paper using a foam brush and the tunnel onset velocity was set to 140 feet/second. The mixture flows under the influence of shear stress and makes patterns on the surface, which indicate the flow topology. The kerosene in the mixture eventually evaporates due to its high volatility and leaves oil traces on the ramp surface. The silica particles thicken the oil so that it does not flow under the effects of gravity when the tunnel flow is off. Then the contact paper was removed from the ramp surface and carefully placed on a flat surface. The visualization images were photographed with a standard digital camera using either UV or white light.

\section{Flow Control Methods}

Steady and unsteady blowing techniques were considered for active flow-control devices in the 2002 study. In addition to the active flow-control techniques, MVGs were used as a passive flow-control device similar to the study in Ref. 4. The MVGs were placed laterally on the ramp shoulder with the trailing edges at the $\mathrm{x}=61.75^{\prime \prime}$ longitudinal station. For steady and unsteady blowing techniques, 0.1175 " diameter circular orifices were used. Sixteen flush-mounted orifices with 0.5 " lateral spacing were fabricated at approximately the same longitudinal station ( $\mathrm{x}=61.75 ")$ into the ramp shoulder (Fig. 2a). The circular round jets (CRJ) have a $30^{\circ}$-pitch angle (angle between jet axis and local surface tangent in the vertical plane). Left-hand-side orifices blow toward the left sidewall and right-hand-side orifices blow toward the right sidewall. These jets have $\pm 90^{\circ}$ azimuthal angle (angle between jet axis and incoming flow in the horizontal plane) to allow symmetric blowing toward the tunnel sidewalls. Due to the azimuthal angle, this type of blowing generates vortices that are similar to the MVG vortices and is referred to as vortex-generating jets in the literature. These vortices deflect the low-momentum flow out toward the tunnel sidewalls and create mixing between upper/lower portions of the boundary layer. The CRJs shared the same plenum and the mass-flow rate to the plenum was kept constant through the use of a flow meter/controller. By opening every other or every third jet orifice, the effect of jet spacing was also studied. The unsteady (pulsed) CRJs were generated by using commercially available high-speed solenoid valves that were placed in the pressure tubing lines between the jet orifice and the plenum. Solenoid valves were connected to a function generator to provide on-demand pulsed jets.

As an active flow-control device, the SWJ actuator was considered the main focus of the 2013 study. The SWJ actuator array was fabricated from acrylic material with a CNC machine and located at $\mathrm{x}=62.325$ " longitudinal station (Fig. 2b). The orifice cross section of the SWJ actuator was square (Aspect Ratio = Width/Depth, AR = 1)

3

American Institute of Aeronautics and Astronautics 
having 0.25 "x 0.25 " dimensions. It should be noted that the actuator orifice is not the same as the actuator exit as shown in Fig. 3a. The actuator orifice is the throat that is slightly upstream of the actuator exit. Different aspect ratio $(\mathrm{AR}=2$ and $\mathrm{AR}=4)$ actuators were obtained by reducing the depth of the actuators to $0.125 "$ and $0.0625 "$ respectively, while maintaining the actuator planform geometry. The SWJ actuator array consisted of five actuators arranged in a lateral line pattern in which the center of the middle actuator coincided with the tunnel centerline (Fig. 2b). Each actuator in the array shared the same plenum. The flow rate to the actuator array was controlled by a flow meter and held constant during the experiment. The SWJ actuator array was placed underneath the ramp and had a jet axis that pointed downstream at an angle of approximately $25^{\circ}$ to the free-stream flow. However since the actuators were placed on the curved surface, the pitch angle between the jet axis and the local flow is $42^{\circ}$. The azimuthal angles of the jets out of SWJ actuator were obtained as $24^{\circ}$ and $34^{\circ}$ by checking the velocity profiles at different downstream locations for angled and oscillatory jets, respectively. The distance between two adjacent SWJ actuators was 2.5". Figure 3a shows the planar view of the SWJ actuator geometry. This figure also explains the working principle of the SWJ actuators. As explained in Refs. 21 and 30, when the fluid passes through the actuator main channel, it attaches to either Coanda surface due to the Coanda effect (Fig. 3a). A backflow develops in the feedback loop and forces the jet flow to detach from that surface and attach to the opposite surface. When the jet flow attaches to the opposite surface, a backflow develops in the other feedback loop, which forces the jet flow to switch back to its initial state. The process then repeats itself, thus producing self-sustained oscillations.

Three different actuation methods were considered in the 2013 study. All three actuation methods used the same SWJ actuators. This allowed direct comparison of the different actuation methods by eliminating many possible disparities in the geometric and actuation parameters, such as location, orientation, spacing, exit area, pressure variations, etc., that may lead to biased conclusions in the comparisons. In the first actuation method, the SWJ actuators were run in a steady (nonoscillating) straight mode. The steady-straight jet (STJ) was generated by using the same actuator with a slight modification as described in Ref. 21. In this modification, the two feedback loops that initiate and maintain the self-sustained oscillations were blocked. In the absence of any backflow from these feedback loops, no oscillation takes place and the jet flow is attached to one of the surfaces inside the actuator due to Coanda effect. In this state, the jet coming out of the actuator exit is steady but has an azimuthal angle between the jet axis and the actuator centerline. In order to eliminate the Coanda effect and hence to generate a nonoscillating straight jet, steady mass flow was provided through the ports to the main jet flow (Fig. 3b). These ports were connected to the inlet nozzle (as shown in Fig. $3 \mathrm{~b}$ as flow line) therefore the total flow rate does not change. The steady mass flow eliminates the jet attachment to the Coanda surfaces and hence produces a nonoscillating straight jet. In this mode, the jet flow out of the SWJ actuator was similar to an axisymmetric turbulent free jet. Since the azimuthal angle was zero, this actuation method produced a straight blowing that energized the boundary layer by momentum addition. In the second actuation method, the SWJ actuators were run in a steady-angled mode. The steady-angled jet was generated by blocking one of the feedback loops as shown in Fig. 3c. A steady backflow develops only in the unblocked feedback loop and this backflow forces the main jet flow always to be attached on the opposite Coanda surface. As a result, the jet flow at the exit was a steady-angled jet. Because of the azimuthal angle, this type of blowing acted as a VGJ that generates streamwise vortices. Note that blocking both feedback loops also generates steady-angled jet. However there is an uncertainty in the sign of the resulting azimuthal angle (either positive or negative) depending on which Coanda surface the main jet flow attaches to inside the actuator. The sign of the azimuthal angle determines whether the generated vortices are clockwise or counter-clockwise rotating vortices. In addition, if the signs of the azimuthal angle of each actuator are the same, then the generated vortices are co-rotating vortices, but if they are mixed then the generated vortices are counter-rotating vortices. Keeping one of the feedback loops open removes this uncertainty and ensures that the jet flow generates the desired type of vortex structures such as, clockwise or counter-clockwise rotating, co-rotating or counter-rotating. In the third actuation method, the SWJ actuators were run in normal operating mode without any modification. This type of blowing generates an oscillating jet at the exit that sweeps back and forth between the two jet-exit sidewalls with a specific frequency. In this current actuator setup the jet out of each actuator oscillates randomly therefore they are not synchronized.

\section{Test Conditions}

\section{A. SWJ Actuators}

First, the SWJ actuator output was characterized by performing bench-top experiments outside the wind tunnel with hot-wire measurements. While the jet flow goes back-and-forth between the two Coanda surfaces inside the SWJ actuator, the resulting jet flow at the SWJ actuator exit is highly oscillatory. In Fig. 4, a typical frequency spectrum of such oscillating flow is given for this type of actuator. The corresponding peak frequency is $160 \mathrm{~Hz}$; however

$$
4
$$

American Institute of Aeronautics and Astronautics 
higher harmonics are also visible in the spectrum. The dominant frequency is very clear and its amplitude is more than any of the other fluctuations. This peak frequency corresponds to the case where the volumetric flow rate was fixed to 13.4 SCFM (standard cubic feet per minute). The hot-wire probe was located downstream of the actuator exit at an arbitrary point ( $6 \mathrm{~mm}$ away from the orifice and $10 \mathrm{~mm}$ off the centerline). Although the amplitude of the spectrum varies depending on the location of the hot-wire probe, the spectrum profile and its peak frequency does not change. The frequency variation of the SWJ actuator with respect to the flow rate was tested by gradually increasing the flow rate and recording the peak frequency (Fig. 5). Consistent with the data available in the literature, ${ }^{24-30}$ the frequency of the actuator varies almost linearly with the flow rate. The oscillation frequency in this study is much lower than the reported frequencies in the literature that are in the $\mathrm{kHz}$ range. This is because the current actuator is much larger than a typical lab-scale actuator of this type reported in the literature. The actuator frequency depends not only on the flow rate but also on the geometry of the actuator. As shown by the other plots in this figure, changing the aspect ratio by reducing the depth of the actuator produced higher frequency responses; however the frequency variations still have linear trends. For the same flow rate (for example, 10 SCFM), the frequencies are $61 \mathrm{~Hz}, 121 \mathrm{~Hz}$ and $252 \mathrm{~Hz}$ for $\mathrm{AR}=1, \mathrm{AR}=2$, and $\mathrm{AR}=4$ actuators, respectively. Since the actuators shared the same plenum, uniformity of each actuator output was also checked for a fixed flow rate. A \pm 5 $\mathrm{Hz}$ deviation was observed in the frequency response from one actuator to the other. This is mostly due to the flow rate variations during the experiments (maximum variation in the flow rate was measured as $1.5 \%$ ). In addition, any geometric mismatch in the fabrication and unequal distribution of the flow to the actuators from the shared plenum can also contribute to the variations in the frequency.

The mean velocity profiles for the three different blowing methods using the SWJ with AR = 2 actuators are shown in Fig. 6. The velocity profiles were nondimensionalized with the average-jet velocity estimated from the flow rate (i.e., $U_{\text {jet }}=Q / A_{\text {jet }}$ ), where $A_{\text {jet }}$ is the total area of jet orifices and $Q$ is the volumetric flow rate. These velocity profiles were measured $20 \mathrm{~mm}$ downstream of the orifice. Since the flow direction and the hot-wire probe axis were not parallel for the oscillating and angled jets, the pitch corrections were applied to the hot-wire measurements to obtain the true velocity as described in Ref. 21. As mentioned before, the straight blowing produces a jet that is similar to an axisymmetric turbulent jet. The peak velocity is at the centerline and the jet half width (spanwise location where the velocity is half the peak velocity) is about $8 \mathrm{~mm}$. the angled blowing produces a VGJ and the velocity profile is similar to the straight jet except that the peak velocity is now shifted (about $9 \mathrm{~mm}$ ) off the centerline. The VGJ velocity profile shows an asymmetric profile where the right tail of the VGJ is longer than the other side. This is because the hot-wire measurements were obtained perpendicular to the actuator centerline rather than the jet axis. The jet half width is very close to that of the straight jet. The oscillatory blowing produces a SWJ in which the jet sweeps back-and-forth between two jet exit sidewalls. As mentioned in Ref. 21, this type of jet produces double peaks and wider spreading. Spanwise locations of the peak velocities of the SWJ and VGJ are very close, which indicates that as the SWJ oscillates, the jet goes from one VGJ state (positive azimuthal angle) to the opposite VGJ state (negative azimuthal angle). The jet half width of the SWJ at the same downstream location is $28 \mathrm{~mm}$, which is more than 3 times larger than that of the nonoscillating jets. As presented in this figure, the increased jet half width is due to the wider spreading, which results in more spanwise coverage. However, continuity of mass implies that the wider spreading results in reduced peak velocities. Even though a reduced peak velocity was observed in the mean velocity profiles, instantaneously the same high-momentum jet affects the outer flow field.

\section{B. Baseline Flow Field}

All of the tests in this study were performed at an onset velocity $\left(\mathrm{V}_{\text {onset }}\right)$ of approximately 140 feet/second. This corresponded to an approximate onset Mach number of 0.12. A boundary-layer trip (grit strip) was applied near the leading edge of the splitter-plate model and at the corresponding streamwise location on the tunnel walls to produce turbulent boundary layers for the onset flow (Fig. 1). Boundary-layer thickness at $x=57$ " station was measured approximately as 0.87 ", which is in good agreement with previously reported data. ${ }^{4}$ Boundary-layer measurements were obtained by custom-made boundary-layer rakes that enabled boundary-layer measurements from 0.007 " above the surface. At the same location, the momentum thickness was obtained as 0.097". The Reynolds number based on the momentum thickness was approximately 6600. The spanwise uniformity of the tunnel flow was checked by comparing the boundary-layer momentum thickness at several spanwise locations between \pm 4.5 " from the tunnel centerline. The maximum variation of the momentum thickness in the spanwise direction was found to be less than $5 \%$ so the free-stream flow upstream of the ramp was assumed to be reasonably uniform.

Typical centerline static-pressure data for the baseline (no flow-control) cases are presented in Fig. 7. As shown in this figure, the baseline cases for the 2002 and 2013 studies match very well, which demonstrates good repeatability over a long time period. For both baseline cases, the $\mathrm{Cp}$ measurements were obtained by taping the

5

American Institute of Aeronautics and Astronautics 
actuator exit holes. The baseline cases have been tested many times to check data repeatability. The maximum standard deviations from the mean Cp were observed mostly at the upstream stations near the suction peaks and measured as $1 \%$ and $0.5 \%$ for the 2002 and 2013 studies, respectively. The centerline Cp distributions show a suction peak at $\mathrm{x}=60$ " station followed by a decrease due to the adverse pressure gradient. The Cp distribution also indicates a flow separation near $\mathrm{x}=64$ " station where a sudden change (plateauing) in the pressure distribution is observed. The centerline suction pressure continues to drop as the separated flow re-attaches to the ramp surface downstream of $\mathrm{x}=70$ " station. The third Cp distribution shown in this plot gives the effect of the jet exits for the 2013 study. Without having any noticeable differences upstream of the separation, there is a slight offset in the Cp distribution, especially in the separated region. As shown in Fig. 2b, the exit shape of the SWJ actuator is trapezoidal. In the absence of tape, the exit holes act as cavities. The two side corners may potentially generate weak counter-rotating vortices. These complex fluid-cavity-vortex interactions may cause an offset in the Cp distribution. The Cp distribution without tape at the exit was used as the baseline (no flow-control) data. The effect of open-jet exits was also checked in 2002 study; however since the exit area of a CRJ is 8 times smaller than the area of the SWJ actuator trapezoidal exit, the effect is very small and within the range of experimental error.

Surface oil-flow visualization for the baseline case is presented in Fig. 8a-b for UV light and white light, respectively. In all oil-flow visualization figures, the flow direction is from top to bottom and the longitudinal $\mathrm{x}$ stations are labelled on the side. This surface flow visualization is very similar to that presented in the Fig. 7 of Ref. 4; however the new surface oil-flow visualization technique provided more detailed flow structures especially in the separated region. In addition, this new visualization technique requires small amounts of mixture (10 ml to cover a 15" x 20" area); therefore it does not lead to excessive collection of oil material, especially in those spiral nodes and tunnel corners. Without having excessive material, the entire surface flow was visualized from one tunnel sidewall to the other including the corner vortices and the reversed flow at the corners. Two large corner vortices were observed on the upper portion of the ramp as shown by two spiral nodes. The separated flow field was slightly biased toward the left-hand side of the figure and this bias was accompanied by a weaker corner vortex at that side. The flow separation and reattachment are seen in Fig. 8. Approximately at $x=64 "$ station, oil material was observed to be collected, which indicates the flow separation location. In these flow visualization images, there is another oil collection site located at $x=67$ " station. Due to the ramp inclination and the presence of reversed flow, the gravity and shear stress forces compete with each other. Therefore this second oil accumulation location can be interpreted as the point where the gravity and the shear stress balance each other. The center of the reattachment node is approximately at $\mathrm{x}=71$ " station. The reattachment locations near the tunnel sidewalls extend further downstream, reaching $\mathrm{x}=74 "$ station. The separated flow is highly three-dimensional because of the interaction between the reversed flow, the corner vortices, and the reattachment node.

\section{Results and Discussions}

\section{A. Active Flow Control with Circular Jet Orifices}

The centerline pressure distribution for the passive flow-control method using MVGs is shown in Fig. 9. The MVG data represents a reference flow-control case for the comparisons. Passive separation control with MVG energizes the boundary layer with addition of convective vorticity. Strong vortices created by MVGs bring highmomentum fluid into the boundary layer and reduces the influence of the corner vortices. The centerline flow remains attached as the suction pressure smoothly decreases under the influence of the adverse pressure gradient. The MVG increased the upstream suction pressure slightly and provided substantial pressure recovery by eliminating the flow separation.

The effect of the steady blowing using the CRJ actuators for different orifice spacing configurations is shown in Fig. 10. The momentum coefficient is $1.1 \%$ as defined by $\quad / \quad$, where $A_{\text {jet }}$ is the total area of jet orifices. $A_{\text {sep }}$ is the surface area of the separated region of the no flow-control case and defined as the product of the tunnel width and the length of the centerline recirculation zone. This length was determined as 8.5 " by investigating the oil-flow visualization images (not shown here) in 2002. Although the new oil-flow visualization technique provided slightly shorter length, the same 8.5" length was used in all cases for consistency. The velocity ratio (VR) is defined as the ratio of the jet velocity to the tunnel onset velocity. The jet velocity was estimated from the flow rate (i.e., $U_{j e t}=Q / A_{j e t}$ ). Three different orifice spacings were studied: 1) 0.5 " spacing with 16 jets, 2) 1" spacing with 8 jets, and 3) 1.5" spacing with 6 jets. These different orifice spacing configurations were obtained by opening every other, or every third jet orifice. Since the number of orifices and hence $A_{\text {jet }}$ was changed, the VR was adjusted to match the same momentum coefficient. By examining the centerline Cp distribution, we did not observe any significant difference due to the orifice spacing as long as the momentum coefficients are the same. This figure 
also indicates that as long as the momentum coefficients are the same, the VR does not have any significant effect on the pressure distribution for the cases tested. Therefore the 8-jet configuration with 1" spacing will be used in the rest of the study. The centerline $\mathrm{Cp}$ distribution indicates that the momentum coefficient has a significant impact on the pressure distribution (Fig. 11). Starting from a low flow rate $(\mathrm{Cm}=0.03 \%$ and $\mathrm{VR}=0.5)$, the blowing rate was gradually increased by an order of magnitude $(\mathrm{Cm}=2.18 \%$ and VR $=4)$. Consistently, we observed a gradually higher upstream suction peak and more pressure recovery at downstream. The steady blowing with $\mathrm{Cm}=0.55 \%$ $(\mathrm{VR}=2)$ resulted in a similar pressure distribution to that of the MVG flow-control case. For the cases tested, $\mathrm{Cm}=$ $0.55 \%$ with the 8 -jet configuration is assumed to be the minimum needed to recover the pressure losses due to the flow separation on the ramp. Therefore the $\mathrm{Cm}=0.55 \%$ will be used as the reference $\mathrm{Cm}$ value. The excessive blowing, $\mathrm{Cm}>0.55 \%$, resulted in additional pressure variations. These favorable Cp distributions were due to the excessive blowing rather than the recovery of the pressure losses. The excessive blowing also reduces the effect of the corner vortices.

Previous investigations have demonstrated that unsteady actuation is more efficient than steady actuation in producing optimal performance. This is because the unsteady actuation requires less energy (or flow rate) input while satisfying the output requirements. ${ }^{8}$ To verify this effect of the unsteady blowing in the current flow-control application, the same 8-jet with $\mathrm{Cm}=0.55 \%$ configuration was used. The momentum coefficient was calculated the same way and mean value of the flow rate was used when calculating the unsteady jet velocity. The unsteady jet actuation was obtained by using high-speed solenoid valves, which were directly connected between the plenum and the orifices. The frequency of the pulsation was varied up to $200 \mathrm{~Hz}$. The frequencies were nondimensionalized by the length of the recirculation zone and the onset velocity to give reduced frequencies (FR) up to 1.00. As shown in Fig. 12, the pressure distribution did not show any dramatic difference. Another set of experiments were also performed (not shown here) to see the effect of unsteady blowing at a lower $C m(0.13 \%)$, which resulted in a similar performance. However, the effect of the unsteady blowing can be revealed by looking more closely at a pressuregradient parameter $(\Delta \mathrm{Cp} / \Delta \mathrm{x})$ that is more sensitive to the flow changes on the ramp model. This parameter is defined to be the difference between a selected lower ramp Cp (at $\mathrm{x}=67.412$ ) and an upper ramp Cp (at $\mathrm{x}=$ 60.125") divided by the distance between their locations. This performance index clearly shows the increased efficiency of the unsteady jet blowing over the steady jet blowing (Fig. 13).

\section{B. Active Flow Control with SWJ Actuators \\ 1. Pressure measurements}

The centerline pressure distribution for the three different blowing methods is shown in Fig. 14. The SWJ actuator with $\mathrm{AR}=1$ was used to produce steady-straight, steady-angled, and unsteady-oscillatory jets. Using the same actuator for both steady and unsteady actuations enables a more direct comparison since it eliminates variations in many actuator parameters (location, orientation, orifice spacing, $\mathrm{A}_{\text {jet }}$, etc.), which may lead to biased conclusions. The flow rate was fixed to 18.6 SCFM to produce the same $\mathrm{Cm}=0.55 \%$. Since the $\mathrm{A}_{\text {jet }}$ of the SWJ actuator is approximately 4 times larger than that of the CRJ orifices, the VR is reduced to 1 (2 for CRJ) for the same $\mathrm{Cm}$. The SWJ actuator array was installed between $\mathrm{x}=61.6^{\prime \prime}$ and $\mathrm{x}=63$ " stations, therefore there is no data points at $\mathrm{x}=62.125 "$ and 62.625" stations in the 2013 study. For comparison, the figure also includes Cp distributions for the no flow-control case and the flow-control case with CRJ from the 2002 study. There is a substantial difference between the no flow-control and flow-control cases for the first two stations right after the blowing exits (stations at 63.4" and 63.9"). This is because those locations were very close to the actuator exits and were exposed to very high-momentum jet flow. All of the flow-control methods produced substantial pressure recovery by reducing the flow separation. Consistently, slightly higher suction peaks were observed on the upstream ramp section. The Cp distributions for the VGJ and SWJ methods are very close to each other in the separated as well as in the upstream ramp regions. Looking more closely at the separated region (Fig. 15) reveals that the SWJ and VGJ are more effective than the STJ by achieving higher pressure recovery and by reducing the flow separation. Although all three methods injected similar momentum into the ramp flow, the superiority of the SWJ and VGJ can be explained by considering the generated streamwise vortices by these (SWJ, VGJ) blowing methods. It should be noted that the STJ blowing method also generates streamwise vortices. However, as shown in the literature, ${ }^{31}$ the circulation level of the vortices generated by the STJ type blowing (zero azimuthal angle) is very small compared to that of an angled jet, i.e. the vorticity generated by the STJ blowing is negligible. The streamwise vortices generated by the SWJ and VGJ methods further energize the boundary layer by bringing the higher momentum fluid toward the ramp surface. As shown in this figure, all three actuation methods (i.e., STJ, VGJ and SWJ) were out performed by the CRJ and the difference between CRJ and the other actuation methods increases gradually for the downstream locations. According to Ref. 31, the streamwise vortex is embedded deeply in the boundary layer and its strength is reduced rapidly for a VGJ with a small velocity ratio. Consequently, due to the reduced velocity (SWJ has VR $=1$

7

American Institute of Aeronautics and Astronautics 
and CRJ has VR = 2), the weaker vortex generated by the SWJ actuation methods decays rapidly in the boundary layer and we observe a gradual deviation in the Cp distributions. Using the CRJ as a point of reference, this deviation means that all three methods (SWJ, VGJ, STJ) are losing their effectiveness on the downstream flow. They are unable to energize the boundary layer by mixing high-momentum fluid from the outer boundary layer as compared to the CRJ case. The weaker vortices do not have enough vorticity at downstream and result in less boundary-layer mixing. As shown in this figure, the momentum-addition method (STJ) and vortex-generating methods (VGJ and SWJ) match very well on the downstream ramp. This supports the conclusion that the generated vortices are weak in the downstream region and only the effect of momentum addition is visible.

Figure 16 shows the effect of actuator aspect ratio on the Cp distribution while focusing only on the separated region. As mentioned before, the aspect ratio was changed by reducing the actuator depth. Three different aspect ratios, $\mathrm{AR}=1, \mathrm{AR}=2, \mathrm{AR}=4$ were tested. In all three cases, $\mathrm{Cm}=0.55 \%$ was fixed by setting the flow rates to 19 , 13.4 and 10 SCFM, respectively. Because of the reduction in the areas, the corresponding velocity ratios were increased to $\mathrm{VR}=1, \mathrm{VR}=1.5$, and $\mathrm{VR}=2$, respectively. As shown in Fig. 5, changing the AR not only changes the VR but also the jet oscillation frequency. For the same $\mathrm{Cm}=0.55 \%$, the SWJ oscillation frequencies are $110 \mathrm{~Hz}$, $163 \mathrm{~Hz}$, and $266 \mathrm{~Hz}$ for $\mathrm{AR}=1, \mathrm{AR}=2, \mathrm{AR}=4$ actuators, respectively. All of the actuation methods produced substantial pressure recovery by reducing the flow separation. The SWJ-AR1 actuator is effective in controlling the flow separation especially in the near exit regions until the $x=67$ " station. Then the Cp distribution deviates from the reference flow-control case (CRJ). This behavior was discussed in Fig. 15 where the vorticity produced by the SWJ-AR1 actuator was weak and decayed earlier reducing its effectiveness in the downstream region. Although a substantial pressure recovery was observed when compared to the no flow-control case, the pressure recovery is less than that of the CRJ actuator. However, it is hard to tell whether it has a separated flow at downstream by simply looking at the centerline Cp distribution. This detail will be discussed by investigating the surface oil-flow visualization in the next section. Increasing the aspect ratio to 2, increased the pressure recovery. Cp distributions of the SWJ-AR2 and CRJ actuators are now matching very well. There is a slight low-offset in the Cp distribution between $\mathrm{x}=65 "-68 "$ stations, which was also observed in SWJ-AR1 case. This slight difference will also be discussed in the surface oil-flow visualizations. Next, the aspect ratio was increased to 4 . This comes with the increased jet velocity (VR = 2), which is the same as that of the CRJ actuator. Interestingly, the AR4 actuator produced less pressure recovery. The reason for this reduced performance of AR4 actuator is unknown, but it may be because the smaller-orifice jet requires a relatively longer distance to penetrate through the boundary layer, although it generates stronger vortex. ${ }^{32}$ Comparing the Cp distributions of the AR1 and AR4 actuators also supports this conclusion. The AR4 actuator is less effective than the AR1 actuator up to $x=69$ " station and more effective afterwards. In addition, the AR4 and AR2 actuators produce similar Cp value after the flow reattachment. Thus, there should be an optimum jet orifice for given jet momentum, orientation, and boundary layer structure. Existence of such optimum orifice was also reported in Ref. 12. We conclude from this figure that the SWJ-AR2 is more efficient than the SWJ-AR1 and SWJ-AR4, and achieves higher pressure recovery by reducing or eliminating the flow separation for the same Cm. Compared to the SWJ-AR1, the SWJ-AR2 requires less flow rate (19 vs. 13.5 SCFM) and provides better performance.

Figure 17 shows the comparison of the steady and unsteady blowing using AR2 actuators. Consistent with the AR1 cases (Fig. 15), the straight blowing is the least effective, which is only energizing the boundary layer by momentum addition. The Cp distributions of the straight blowing for AR1 and AR2 are very similar. This indicates that changing VR $=1$ to VR $=1.5$ does not provide any noticeable difference in the Cp distributions for the straight blowing as long as the $\mathrm{Cm}$ is the same. As previously discussed in Fig. 15, the VGJ-AR2 actuator further increased the pressure recovery in the entire separated flow region by distributing the high-momentum fluid in the boundary layer through mixing. The VGJ-AR2 actuator produces very similar Cp distribution to that of the VGJ-AR1 actuator in the near exit region until the $\mathrm{x}=68$ " station, and then it provides more pressure recovery at the downstream stations. This also supports the previous conclusions that the AR1 actuators generate weaker vortices. Since the AR2 actuator has VR $=1.5$, the vortex generated by AR2 actuators is stronger than that of AR1 actuators. Therefore the stronger vortices survive longer and penetrate further into the downstream stations resulting in better performance. While the VGJ-AR1 and SWJ-AR1 actuators produced very similar Cp distributions (Fig. 15), the SWJ-AR2 actuator produced more pressure recovery than the VGJ-AR2 especially between the $x=65 "-70$ "stations. We do not see any difference after the station $70 "$ due to the attached flow. Therefore for the cases tested, the oscillatory blowing with SWJ-AR2 is considered the most effective.

Usually the $\mathrm{Cm}$ value is the most important parameter in the blowing type active flow-control methods. This is because adding momentum to the boundary layer directly increases near-wall fluid momentum and thus reduces or completely eliminates the flow separation. In addition, for a fixed geometry, higher $\mathrm{Cm}$ results in higher VR, which generates a stronger vortex. The effect of the $\mathrm{Cm}$ value was tested by gradually increasing the flow rate. As observed

8

American Institute of Aeronautics and Astronautics 
with the $\mathrm{Cm}$ variation in the CRJ blowing method (Fig. 11), increasing the $\mathrm{Cm}$ has a favorable effect on the Cp distribution by increasing both the downstream pressure recovery and upstream suction peak (Fig. 18). Since the flow is already attached for $\mathrm{Cm}=0.55 \%$, further increasing the $\mathrm{Cm}$ value has more effect on the downstream flow, that is due to the excessive blowing rather than the pressure recovery. Another set of experiments was performed with AR1 and AR2 to investigate the effect of vorticity strength by increasing the $\mathrm{Cm}$ to $1.2 \%$. The corresponding VR values are 1.57 and 2.2, (oscillation frequencies are $157 \mathrm{~Hz}$ and $210 \mathrm{~Hz}$ ) for the AR1 and AR2 actuators, respectively. Since only VGJ and SWJ type blowing generate streamwise vortices, the straight blowing results were not included in Fig. 19. In addition, the $\mathrm{Cm}$ of the reference flow-control case (CRJ, $\mathrm{Cm}=0.55 \%$.) was kept the same. It is observed that increasing the vorticity strength by increasing the VR $=1$ to $\mathrm{VR}=1.57$, improves the performance of the AR1 actuator. Cp distributions of the AR1 actuators are either matching or better than that of the CRJ actuators (Fig. 19, compare with Fig 14). This demonstrates that it recovers the pressure losses due to the adverse pressure gradient, and hence eliminates the flow separation in the entire region. Interestingly, the $\mathrm{Cp}$ distributions of the VGJ-AR1 and SWJ-AR1 match each other similar to the $\mathrm{Cm}=0.55 \%$ case in Fig. 15 . However, increasing the vorticity strength by increasing the VR $=1.5$ to $\mathrm{VR}=2.2$ for AR2 actuator does not show a substantial difference. This is consistent with the CRJ results presented in Fig. 10, where changing VR does not have any significant effect on the pressure distribution as long as the momentum coefficients are the same. Increasing $\mathrm{Cm}$ to $1.2 \%$ produced very similar $\mathrm{Cp}$ distributions with that of $\mathrm{Cm}=0.55 \%$ for both SWJ-AR2 and VGJ-AR2 actuators. Therefore we can conclude that at least VR $=1.5$ is required for AR1 and AR2 actuators to recover the pressure losses due to the flow separation. Once the flow separation is eliminated, further increasing the $\mathrm{Cm}$ only generates favorable $\mathrm{Cp}$ distribution due to excessive blowing.

Figure 20 shows the comparison of two different vortex-generating jets (generated by CRJ and SWJ actuators) at two different $\mathrm{Cm}$ values. We have observed in the previous figures that the SWJ actuators either provide similar Cp distributions to that of the CRJ blowing or were out-performed by the CRJ blowing. One of the reasons may be the slight difference in the locations of the CRJ and the SWJ exits, where the CRJ exits were placed about 0.5" upstream of the SWJ actuators location due to the space limitations. This extra distance allows the CRJ-generated vortices better mixing and deeper penetration into the boundary layer. The other reason may be due to the difference in the azimuthal angle, which has been found to be an important parameter to achieve the desired separation control. ${ }^{6}$ It was reported that although a small azimuthal angle is preferred for the rectangular slot jets, a large azimuthal angle is more effective for the circular orifice jets. ${ }^{33}$ There are mixed results for the optimum azimuthal angle in the literature and these results are very much dependent on the flow conditions, but the azimuthal angle of $90^{\circ}$ was reported to be well-suited to prevent the flow separation on an adverse-pressure-gradient ramp. ${ }^{34}$ Therefore, the CRJ actuators have the optimum $90^{\circ}$ azimuthal angle, whereas the $24^{\circ}$ of azimuthal angle may not be the optimum for the VGJ generated by the SWJ actuator. When we compare the effects of two vortex generator methods, they both provided substantial downstream pressure recovery by reducing the flow separation. Increasing the $\mathrm{Cm}$ has a favorable effect on the downstream pressure recovery. Interestingly, while increasing $\mathrm{Cm}$ did not result in any significant difference in the upstream suction pressures for the VGJ with SWJ actuator, we see a large increment in the case of CRJ. This can be explained by considering the actuation of each vortex generator method. The VGJ with SWJ actuators are mainly acting on the separated flow between two corner vortices. Their interaction increases the upstream suction pressures by eliminating the flow separation. Once the flow separation is eliminated, increasing $\mathrm{Cm}$ does not increase the upstream suction pressures although there is slightly more downstream pressure recovery due to excessive blowing. On the other hand, the CRJ blowing affects the separated flow in a different manner. First of all, the CRJ orifices were oriented such that four left-hand side orifices blow toward tunnel-left sidewall, producing co-rotating clockwise vortices (looking upstream). We hypnotized that these co-rotating vortices merge together to form a single large and stronger clockwise rotating vortex. ${ }^{6,35}$ On the opposite side, four right-hand side orifices blow toward the tunnel-right sidewall, producing counter-clockwise co-rotating vortices. These vortices also merge together to form a single large and stronger counter-clockwise rotating vortex that is symmetric to the clockwise-rotating vortex. These two vortices form one large counter-rotating vortex pair covering the tunnel test section. A relatively narrow tunnel test section also contributes in the formation of the large counter-rotating vortex pair. The counter-rotating vortex pair is in common flow down configuration with its maximum downwash region is along the centerline where the pressure ports are located. As demonstrated in Ref. 7, the counter-rotating vortices generate spanwise variation in the streamwise pressure distributions with its maximum performance along the maximum downwash region. The side flow induced by the counter-rotating vortex deflects the lower momentum flow outward from the centerline and produces locally thinned boundary layer. In addition, the large counter-rotating vortex pair has more influence on the corner vortices. Even after the flow separation is eliminated on the ramp, the large counter-rotating vortex pair further increases the suction pressure by reducing the effect of corner vortices.

9

American Institute of Aeronautics and Astronautics 


\section{Surface oil flow visualizations}

$\mathrm{Cp}$ measurements in the previous sections only show the effect of the active flow-control techniques on the centerline locations and do not show what's happening on the ramp surface. Even if it is possible to deduce the separation location by checking the sudden changes (such as plateauing) in the pressure distribution, this is not practical for every spanwise location. As shown in the previous $\mathrm{Cp}$ measurements, the active flow-control techniques recovered some of the pressure losses by reducing the centerline flow separation, but it is unknown whether the flow separation is eliminated across the ramp. Surface oil-flow visualization is a valuable tool that shows the flow topology on the surface of interest. Even though surface oil-flow visualization is a qualitative technique, it has been extensively used to observe flow separation and reattachment locations. Because the separated flow field is three-dimensional, the oil-flow visualizations not only indicate the separation and reattachment locations, but also give information about the surface flow topology around the flow separation. In addition, since the focus of this study was to investigate different active flow-control techniques, the effects of these actuation methods and their performance evaluations are also provided. Surface flow visualization will be used to fully explain the centerline $\mathrm{Cp}$ distributions. Surface oil-flow visualization for the no flow-control case was previously given in Fig. 8a-b. Using the newly developed oil-flow visualization technique, we were able to clearly see the three-dimensional flow separation over the ramp. Two corner vortices together with the reattachment node were presented. It was shown that the flow separates at $x=64$ " station and reattaches to the ramp surface at $x=71 "$ station. The reversed flow between separation and reattachment locations was clearly demonstrated. Because of the three-dimensional flow separation and the corner vortices, the reattachment location was a node rather than a line. It has been shown in the reference flow-control case that $\mathrm{Cm}=0.55 \%$ is enough to recover the pressure losses, therefore we only consider the $\mathrm{Cm}=0.55 \%$ in the flow visualizations. In addition, since the AR2 actuator achieved the best performance at this specific $\mathrm{Cm}$, the flow visualization results with the AR2 actuators will be presented. However, for selected cases, we will also present results with different $\mathrm{Cm}(\mathrm{Cm}=1.2 \%)$ and different aspect ratio (AR1) to compare the effects of the momentum coefficient, the aspect ratio and the velocity ratio on the separation control using SWJ actuators.

Figure 21 shows the surface oil-flow visualization of the flow separation control using the straight-blowing method with the STJ-AR2 actuators. Red arrows (2.5" apart) in this figure represent the spanwise locations of the five actuators that blow steady-straight jet directly downstream. When compared with the no flow-control case (Fig. 8), a different flow topology was observed. Instead of clear reversed flow patterns, stagnant oil material was observed on the ramp surface between two corner vortices due to the low shear stress. The stagnant oil material was observed to start at $\mathrm{x}=64$ " station where the flow separates in the no flow-control case (Fig. 8) and extend to $\mathrm{x}=$ 70"-73" downstream stations. In the spanwise direction, the low shear stress region extends \pm 2.5 " away from the centerline. This low shear stress region can be interpreted as the separated flow region. As given in the corresponding Cp distribution (Fig. 17), there is a substantial recovery of the pressure losses by addition of linear momentum into the boundary layer, however compared to the reference flow-control case, the STJ blowing did not completely recover the pressure losses and did not eliminate the flow separation. A clear interaction between the reversed flow and the corner vortices was shown previously in no flow-control case (Fig. 8). Due to the interactions observed in Fig. 8, we saw not only a reversed flow, but also lateral flow that pooled oil from the centerline to the tunnel sidewalls in the separated flow region. However in Fig. 21, the flow separation is reduced and the corner vortices are restrained toward the sides so that we do not see a clear interaction between the separated flow and the corner vortices near the surface. Another difference from the no flow-control case is that we no longer see a reattachment node; instead we see reattachment points that are not uniform in the spanwise direction. For example, the reattachment location near the centerline (downstream of the middle actuator), is approximately at $\mathrm{x}=70 "$ station; while it extends to $\mathrm{x}=72.5$ " station between two actuators. Although the strength of the recirculating flow is reduced and both the location and the structure of the reattachment are changed, the location of the flow separation is not changed indicating no flow separation delay.

In the next figure (Fig. 22), surface oil-flow visualization of the separation control using angled blowing with VGJ-AR2 actuators is shown. The same actuator and flow rate $(\mathrm{Cm}=0.55 \%)$ were used. Red arrows in this figure represent the spanwise locations of the actuator exits as well as the blowing direction. Actuators provided a steadyblowing jet that was angled toward the left-hand side. The interaction of the angled jet with the onset tunnel flow generated clockwise rotating streamwise vortices. Since all of five actuators blew in the same direction, the generated vortices were co-rotating. These co-rotating streamwise vortices are depicted as red curved arrows in the figure. One vortex was generated per actuator so there are five co-rotating vortices in the domain. Only three corotating vortices are shown in Fig. 22 because the two outside vortices are swallowed by the two corner vortices. As the vortices travel downstream, they expand and lose their strength by exchanging momentum within the boundary layer. These streamwise vortices help to recover the pressure losses that are caused by the adverse pressure gradient.

10

American Institute of Aeronautics and Astronautics 
As seen in this oil-flow visualization, the generated vortices further reduced the flow separation region when compared to the straight blowing case. Because both of these actuation methods used the same actuator and the same $\mathrm{Cm}$, this extra performance is due to the generated streamwise vortices. Similar stagnant oil material was observed at $\mathrm{x}=64$ " station, which indicates the flow separation. Similar to the STJ blowing, the separation location does not seem to be affected. Due to the left-hand side blowing, we see an increased bias of the ramp flow field toward the left-hand side of the figure that is accompanied by a smaller corner vortex on that side. At the separation line, we can clearly see lateral flow patterns towards the left-hand side. The generated streamwise vortices effect only a small region (about 1 " wide, see downstream of middle actuator) due to the vortex's small size at the beginning; however as it travels downstream, it expands and meets with the nearby vortices at about station 70.5", indicating 2.5" wide effective area. As seen in this figure, three-streamwise vortices cover the tunnel in the spanwise direction. The VGJ produced two separated flow regions that were about 1.5" wide instead of one big separated region (5" wide) as in the straight-blowing case. Reattachment location is at $x=69$ " station compared to the $x=70 "-72.5$ " stations for the STJ. The area of the separated region was roughly calculated by applying simple image processing technique. The comparison of the separated region with that of the STJ blowing reveals more than $50 \%$ reduction in the separated area (32 vs. 14 inch $^{2}$ ). This performance increase is also consistent with the corresponding Cp distribution (Fig. 17).

Figure 23 shows the surface oil-flow visualization of the separation control using the oscillatory blowing with SWJ-AR2 actuators. Since the oscillatory jet is going back-and-forth between two jet-exit sidewalls, the effect of SWJ actuator can be explained by making a similar analogy with the steady VGJ effect. However, since the oil-flow visualization (also Cp measurements) shows only the mean effect, this analogy is only true when explaining the mean effect of the unsteady SWJ actuators. As reported in the literature, ${ }^{21}$ the sweeping motion of the jet from one side to another produces a gusty flow field. This gusty flow field carries vortices of different sizes and strengths, which may be very beneficial in the boundary-layer mixing process and for controlling the flow separation. However, the measurement techniques used in this study (Cp measurements and flow visualizations) only allow us to draw conclusions about the mean effect of the SWJ actuators. It has been reported ${ }^{21}$ that the jet spends more time on each Coanda surface producing a left-hand side blowing VGJ in the first half period and right-hand side blowing VGJ in the second half period. Momentarily, the SWJ produces a clockwise-rotating vortex when the jet is on the left-hand side and then generates counter-clockwise rotating vortex when the jet is on the right-hand side. Two counter-rotating vortices are generated per actuator. Since these counter-rotating vortices are generated at two consecutive half-periods, there is a phase difference between them (half-period) and they do not cancel each other's effect. Two counter-rotating vortices generated by the same actuator are in common flow down configuration that brings the higher-momentum fluid from the outer boundary layer toward the wall. These common-flow-down vortices generate $\boldsymbol{\Lambda}$-shaped flow patterns along the actuator centerlines. On the other hand, the two counter-rotating vortices from two adjacent actuators are in common flow up configuration that causes the local ramp flow to lift off the surface forming longitudinal flow detachment lines between two actuators. When compared with the VGJ method where only one vortex is generated per actuator and the vortex affects only one side of the actuator centerline, the SWJ method generates two vortices per actuator that affect both sides of the actuator centerline. Therefore we see an increased coverage for the oscillatory blowing with SWJ actuator even though all the actuator parameters are the same. As we see in the oil-flow visualization figure, the separated flow region is now two small islands between two actuators. The separation location is at $\mathrm{x}=64$ " station and the flow reattachment is about $\mathrm{x}=$ $65.5 "$ station. The calculated areas reveal that the SWJ actuators reduce the separated region almost 3 times compare to the VGJ actuators (areas are 5 vs. 14 inch $^{2}$ ) and more than 6 times compare to the STJ actuators (5 vs. 32 inch $^{2}$ ). This is also consistent with the centerline Cp distribution in Fig. 17. As shown in that figure, the SWJ method produces better performance between $\mathrm{x}=64 "-69$ " stations. When we look at the oil-flow visualization figure of VGJ (Fig. 22), we noticed a separated flow region between $x=64 "-69 "$ stations. However, for the oscillatory-blowing case, the flow separation is restricted to two small islands between $\mathrm{x}=64 "-65.5$ " stations. These oil-flow visualizations help to explain the different Cp distributions with different actuation methods.

Increasing the momentum coefficient to $\mathrm{Cm}=1.2 \%$ did not provide any substantial difference in the flow visualization (Fig. 24). This is because the flow is already attached in the majority of the domain, and blowing harder only adds more momentum to the boundary layer. Without any pressure losses due to the flow separation, further energizing the boundary layer only results in slightly better Cp distribution as we saw in Fig. 18. Another flow visualization experiment was performed to see the effect of changing the orifice aspect ratio. For this purpose, SWJ-AR1 actuators were installed and run at the same $\mathrm{Cm}$ (0.55\%). The oil-flow visualization in Fig. 25 does not reveal any substantial difference. We see attached flow in the majority of the ramp flow; there are two small islands of separated flow region as similar with SWJ-AR2 actuators in Fig. 23. We can clearly see some oil accumulation between two actuators where the counter-rotating vortices form a lift off flow. As we go back to Fig. 16, the Cp distribution of the SWJ-AR1 actuators was matched very well until $\mathrm{x}=67$ " station and then deviated from that of

11

American Institute of Aeronautics and Astronautics 
the SWJ-AR2 that resulted in less pressure recovery. Although producing less pressure recovery, this figure indicates that the SWJ-AR1 actuator at $\mathrm{Cm}=0.55 \%$ is also effective in controlling the flow separation. Since the SWJ-AR1 actuator requires more flow rate for a fixed $\mathrm{Cm}$ than the SWJ-AR2 (19 vs. 13.4 SCFM), the SWJ-AR2 actuators are more efficient than the SWJ-AR1 actuators. In another experiment, the momentum coefficient of the SWJ-AR1 was increased to $\mathrm{Cm}=1.2 \%$ (Fig. 26). Increasing the $\mathrm{Cm}$ resulted in an increase in the vortex strength as discussed previously, which resulted in completely attached flow in the entire domain. The two small islands of flow separation that were present in both SWJ-AR1 $(\mathrm{Cm}=0.55 \%)$ and SWJ-AR2 $(\mathrm{Cm}=1.2 \%)$ actuations were now completely eliminated. Consistent with the Cp measurements (Fig. 19), the SWJ-AR1 actuation resulted in the best performance by eliminating those small separated flow regions. However, since the flow is already attached after $\mathrm{x}=$ $66 "$ station, we do not see any noticeable difference after the reattachment. The only difference when compared with the SWJ-AR1 with $\mathrm{Cm}=0.55 \%$ is that since the generated vortices are stronger than that of the $\mathrm{Cm}=0.55 \%$ case, we now clearly see the longitudinal vortex lift-off lines between two adjacent actuators.

\section{Conclusions}

Various flow separation control methods were investigated on an adverse-pressure-gradient ramp model. Methods to characterize and compare different types of flow-control actuation were developed. The results were presented in the form of centerline $\mathrm{Cp}$ measurements and oil-flow visualizations. A new surface oil-flow visualization technique was developed that enhanced the details of the flows observed in this study. The technique has the advantages of the kerosene $+\mathrm{TiO}_{2}$ and the fluorescent oil-film techniques. Three-dimensional separated flow, corner vortices, and the reattachment nodes/points were clearly shown with this new technique.

The experimental data in this study consist of two different data sets acquired during two different time periods. The first data set includes active and passive separation control techniques. Passive separation control with MVG energizes the boundary layer with addition of convective vorticity. Strong vortices created by MVGs bring highmomentum fluid into the boundary layer and reduce the influence of the corner vortices. MVGs provide substantial pressure recovery by eliminating the flow separation. The pressure distribution of MVG was used to determine the required momentum coefficient for the active flow control methods. Active separation control was performed in the form of both steady and unsteady blowing through circular jet orifices. First, the effect of orifice spacing was studied. It was found that as long as the momentum coefficients are the same, changing the orifice spacing has negligible effect. This is also true for the velocity ratio, but only after a certain threshold level. On the other hand, the momentum coefficient has a significant effect on the flow separation control. Increasing the momentum coefficient always results in higher suction peaks and more pressure recovery even after the flow is completely attached. Unsteady actuation was accomplished by using solenoid valves. Even though the Cp distribution did not reveal the unsteady effects, a pressure gradient term clearly showed that unsteady blowing is more effective than steady blowing.

The second data set focused on the SWJ actuators and their different forms of blowing configurations. The SWJ actuators were run in three different modes to produce steady-straight (STJ), steady-angled (VGJ), and unsteadyoscillating jets (SWJ). Centerline Cp measurements and oil-flow visualizations were provided. Actuation methods, $\mathrm{Cm}, \mathrm{AR}$, and VR are the parameters that were investigated. It was found that the STJ blowing is the least effective method for a fixed $\mathrm{Cm}$. This is because the STJ blowing energizes the boundary layer primarily by momentum addition. Although the STJ blowing provides substantial pressure recovery compared to the no flow-control case, the surface flow visualization reveals that the flow is still separated. The VGJ blowing achieves better performance than the STJ. This is because the VGJ generates streamwise vortices that energize the boundary layer by bringing highmomentum fluid into the boundary layer. Surface flow visualization of VGJ shows that one clockwise-rotating vortex per actuator is generated. These streamwise co-rotating vortices reduce the separated region more than $50 \%$ compared to the STJ blowing. The oscillatory blowing achieves the best performance through increased pressure recovery and reduced flow separation region. SWJ flow visualizations indicate that two counter-rotating vortices are generated per actuator. The two counter-rotating vortices are generated by the same actuator at different phases of the oscillation cycle; therefore they are out of phase and do not cancel each other's effect. The SWJ influences more area, because of the two counter-rotating vortices. Increased coverage results in more than 3 and 6 times reduction in the separated flow area compared to the VGJ and STJ methods, respectively. The SWJ-AR1 actuator has inferior performance when compared to the SWJ-AR2 actuator. This is caused by the weaker vorticity production due to the

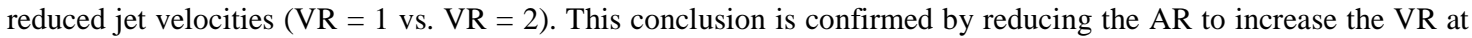
the same $\mathrm{Cm}$. The $\mathrm{AR}=2$ actuator provides the best flow-control performance. Interestingly, further increasing the AR ( $\mathrm{AR}=4$ ) did not provide any improvements even though the $\mathrm{Cm}$ is the same and the VR is higher (stronger vortex). This reduced performance is attributed to the longer distance requirement of the smaller-orifice jets to

12

American Institute of Aeronautics and Astronautics 
penetrate through the boundary layer. The effect of vorticity strength was tested by doubling the momentum coefficient with $\mathrm{AR}=1$ and $\mathrm{AR}=2$ actuators. Doubling the $C m$ increases the VR to 1.5 from 1 for $\mathrm{AR}=1$ actuators and to 2.2 from 1.5 for $\mathrm{AR}=2$ actuators. Doubling the $\mathrm{Cm}$ improved the performance of the $\mathrm{AR}=1$ actuator by increasing the pressure recovery as shown in the Cp measurement and eliminating the flow separation as shown in the surface flow visualizations. However, doubling the momentum coefficient of $A R=2$ actuator did not provide any better results. The $\mathrm{Cp}$ distribution and the flow visualization images of both $\mathrm{Cm}$ values are very similar. In terms of velocity ratio, while increasing the VR to 1.5 from 1 results in better performance, increasing the VR to 2.2 from 1.5 does not show any significant difference. This is explained by the existence of a threshold limit for VR, found as 1.5 for the current configurations tested, after which the SWJ is effective.

Comparison of the SWJ and CRJ actuators demonstrates that the SWJ actuator provides either similar Cp distributions to that of the CRJ actuator or is out-performed by the CRJ actuator. It was concluded that the CRJ actuators have the optimum azimuthal angle. In addition, because of the orientation of the CRJ orifices, the generated vortices can merge together to form a large counter-rotating vortex pair with its maximum downwash region is along the centerline where the pressure ports are located. The side flow induced by this vortex pair deflects the lower momentum flow outward from the centerline and produces a locally thinned boundary layer. Due to this large vortex pair, the CRJ actuator also has more influence on the corner vortices, which was explained by looking at the suction pressures before the separation location. While increasing the momentum coefficient does not increase the upstream suction pressures once the flow is attached for the SWJ actuators, it gradually increases the upstream suction pressures for the CRJ actuators by reducing the effect of corner vortices. The CRJ actuators are optimized for the given flow-control application but the SWJ actuators are more adaptive to the changing flow conditions that make them attractive flow-control actuators. Furthermore, when we remove the advantages that the CRJ actuators have on the SWJ actuators by making a more direct comparison between the SWJ actuator operated in either steady or unsteady mode, we find that the unsteady SWJ outperforms the steady VGJ. This would make the unsteady SWJ the preferred flow-control method.

\section{Acknowledgment}

The study of the first author is supported by the Fixed Wing Project of NASA's Fundamental Aeronautics Program. The study of the second author was supported by the former NASA UEET Program. The authors would like to thank the following individuals for their input: Rudolph King, John Lin, Frank Chen, and Charlie Debro.

\section{References:}

30.

${ }^{1}$ Gad-el-Hak M., Bushnell D. M., “Separation Control: Review”, Journal of Fluids Engineering, Vol.113, No.1, 1991, pp.5-

${ }^{2}$ Schubauer G. B., Spangenber W. G., "Forced mixing in boundary layers", Journal of Fluid Mechanics, Vo. 8, No. 1, 1960, pp. 10-32.

${ }^{3}$ Lin J. C., Howard F. G., Selby G. V., "Small submerged vortex generators for turbulent flow separation control”, Journal of Spacecraft and Rockets, Vol. 27, No. 5, 1990, pp.503-507.

${ }^{4}$ Jenkins L., Gorton S. A., Anders S., "Flow control device evaluation for an internal flow with an adverse pressure gradient", AIAA Paper 2002-0266, January 2002.

${ }^{5}$ McCormick D. C., "Shock-boundary layer interaction control with low-profile vortex generators and passive cavity" AIAA Paper 92-0064, January 1992.

${ }^{6} \mathrm{Lin}$ J. C., "Review of research on low-profile vortex generators to control boundary-layer separation," Progress in Aerospace Sciences, Vol.38, 2002, pp. 389-420

${ }^{7}$ Selby, G.V., Lin, J.C., and Howard, F.G., "Control of Low-speed Turbulent Separated Flow Using Jet Vortex Generators," Experiments in Fluids, Vol. 12, 394-600, 1992

${ }^{8}$ Greenblatt, D. and Wygnanski, I. J., "The control of flow separation by periodic excitation," Progress in Aerospace Sciences, Vol. 36, No.7, 2000, pp. 487-545,

${ }^{9}$ McManus, K. R., Legner, H. H., and Davis, S. J., "Pulsed Vortex Generator Jets for Active Control of Flow Separation," AIAA paper 94-2218, June 1994.

${ }^{10}$ Tilmann, C. P., Langan, K. J., Betterton, J. G., and Wilson, M. J., “Characterization of Pulsed Vortex Generator Jets for Active Flow Control," Presented at the RTO AVT Symposium on Active Control Technology for Enhanced Performance Operation Capabilities of Military Aircraft, Land Vehicles and Sea Vehicles, Germany, May 2000.

${ }^{11}$ Johari, H., and Rixon, G. S. "Effects of pulsing on a vortex generator jet." AIAA Journal, Vol. 41, No. 12, 2003, pp. 23092315.

${ }^{12}$ McManus, K., Ducharme, A., Goldey, C., and Magill, J., 1996, "Pulsed Jet Actuators for Suppressing Flow Separation," AIAA Paper 96-0442 
${ }^{13}$ Roth, J. R., Sherman, D. M., and Wilkinson, S. P., "Electrohydrodynamic flow control with a glow-discharge surface plasma," AIAA Journal, Vol. 38, No. 7, 2000, pp. 1166-1172.

${ }^{14}$ Enloe, C. L., McLaughlin, T. E., VanDyken, R. D., Kachner, K. D., Jumper, E. J., Corke, T. C., Post, M., and Haddad, O., "Mechanisms and Responses of a Single Dielectric Barrier Plasma Actuator: Geometric Effects," AIAA Journal, Vol. 42, No. 3, 2004, pp. 595-604.

${ }^{15}$ Glezer, A. and Amitay, M., "Synthetic jets" Annual Review of Fluid Mechanics, Vol. 34, 2002, pp. 503-529.

${ }^{16}$ Viets H. "Flip-flop jet nozzle". AIAA Journal, Vol. 13, 1975, pp.1375-1379

${ }^{17}$ Stouffer, R. D., "Liquid Oscillator Device", U.S. Patent 4,508,267, Issued April 2, 1985.

${ }^{18}$ Beale, R. B. and Lawler, M. T., "Development of a wall-attachment fluidic oscillator applied to volume flow metering," Flow: Its measurement and control in science and industry. Vol. 1, ISA, 1974, pp. 989-996.

${ }^{19}$ Wang, H., Beck, S. B. M., Priestman, G. H., and Boucher, R. F., "Fluidic pressure pulse transmitting flowmeter," Chemical Engineering Research \& Design, Transactions of the Institute of Chemical Engineers, Part A, Vol. 75, No. A4, 1997, pp. 381-391.

${ }^{20}$ Vatsa, V., Koklu, M., and Wygnanski, I., "Numerical Simulation of Fluidic Actuators for Flow Control Applications", AIAA paper 2012-3239, June 2012.

${ }^{21}$ Koklu, M., and Melton, L.P., "Sweeping Jet Actuator in a Quiescent Environment ", AIAA 2013-2477, June 2013.

${ }^{22}$ Bobusch, B. C., Woszidlo, R., Bergada, J. M., Nayeri, C. N., and Paschereit, C. O., "Experimental Study of the Internal Flow Structures inside a Fluidic Oscillator," Experiments in Fluids, Vol. 54, No. 6, 2013.

${ }^{23}$ Gaertlein, S., Woszidlo, R., Ostermann, F., Nayeri, C.N., \& Paschereit, C.O., "The Time-Resolved Internal and External Flow Field Properties of a Fluidic Oscillator,” AIAA 2014-1143 January 2014

${ }^{24}$ DeSalvo, M., Whalen, E., and Glezer, A., "High-Lift Enhancement using Fluidic Actuation,” AIAA 2010-0863, January 2010

${ }^{25}$ Seele, R., Tewes, P., Woszidlo, R., McVeigh, M. A., Lucas, N. J., and Wygnanski, I. J., “Discrete Sweeping Jets as Tools for Improving the Performance of the V-22," Journal of Aircraft, Vol. 46, No. 6, Nov.-Dec. 2009.

${ }^{26}$ Cerretelli, C., Wuerz, W., and Gharaibah, E., "Unsteady Separation Control on Wind Turbine Blades Using Fluidic Oscillators," AIAA Journal, Vol. 48, No. 7, 2010, pp. 1302-1311.

${ }^{27}$ Seifert, A., Stalnov, O., Sperber, D.,Arwartz, G., Palei, V.,Davis, S., Dayan, I., and Fono, I., "Large Trucks Drag Reduction Using Active Flow Control", AIAA 2008-0743, January 2008.

${ }^{28}$ Woszidlo, R., Stumper, T., Nayeri, C. N., and Paschereit, C. O., "Experimental Study on Bluff Body Drag Reduction with Fluidic Oscillators," AIAA 2014-0403, January 2014

${ }^{29}$ Seele R., Graff, E., Gharib, M., Taubert, L., Lin, J., and Wygnanski, I. "Improving Rudder Effectiveness with Sweeping Jet Actuators", AIAA paper 2012-3244, June 2012

${ }^{30}$ Raman, G., and Raghu, S., "Cavity Resonance Suppression Using Miniature Fluidic Oscillators," AIAA Journal, Vol. 42, No. 12, 2004, pp. 2608-2612

${ }^{31}$ Zhang, X., "Co- and Contrarotating Streamwise Vortices in a Turbulent Boundary Layer," Journal of Aircraft, Vol. 32, No. 5, 1995, pp. 1095-1101.

${ }^{32}$ Rixon, G. S., and Johari, H., "Development of a Steady Vortex Generator Jet in a Turbulent Boundary Layer," Journal of Fluids Engineering, Vol. 125, 2003, pp. 1006-1015

${ }^{33}$ Casper, M., and Kähler, C. J., and Radespiel, R., "Fundamentals of Boundary Layer Control with Vortex Generator Jet Arrays," 4th AIAA Flow Control Conference, AIAA Paper 2008-3995, 2008.

${ }^{34}$ von Stillfried, F., Wallin, S., and Johansson, A. V.,"Evaluation of a Vortex Generator Model in Adverse Pressure Gradient Boundary Layers,” AIAA Journal , Vol. 49, No. 5, 2011, pp. 982 -993

${ }^{35}$ Marles, D. and Gursul, I., "Effect of a Jet on Vortex Merging," 37th AIAA Fluid Dynamics Conference and Exhibit, AIAA 2007-4364, June 2007 


\section{Figures}

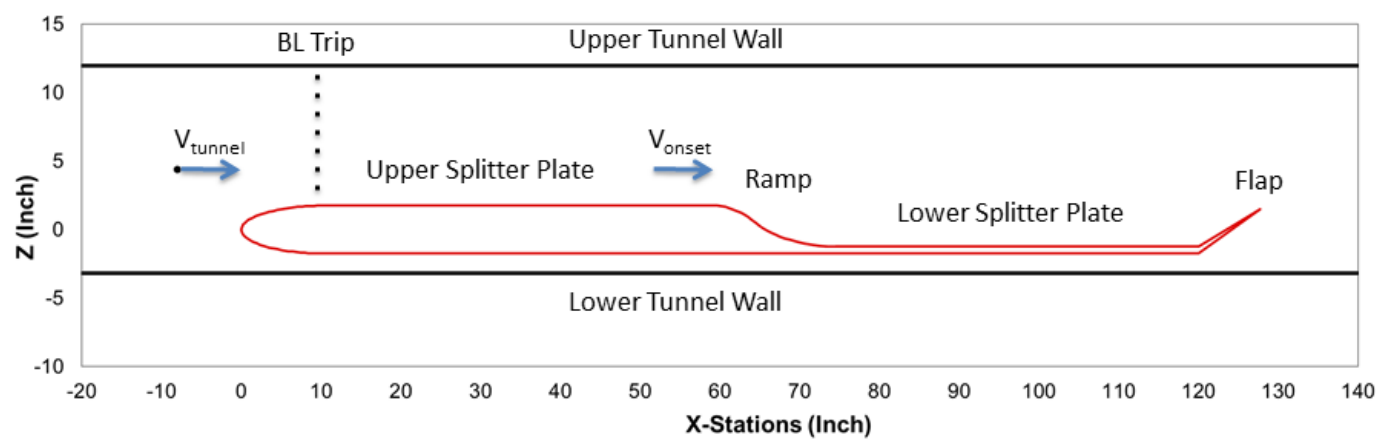

Figure 1. General diagram of the wind-tunnel configuration.
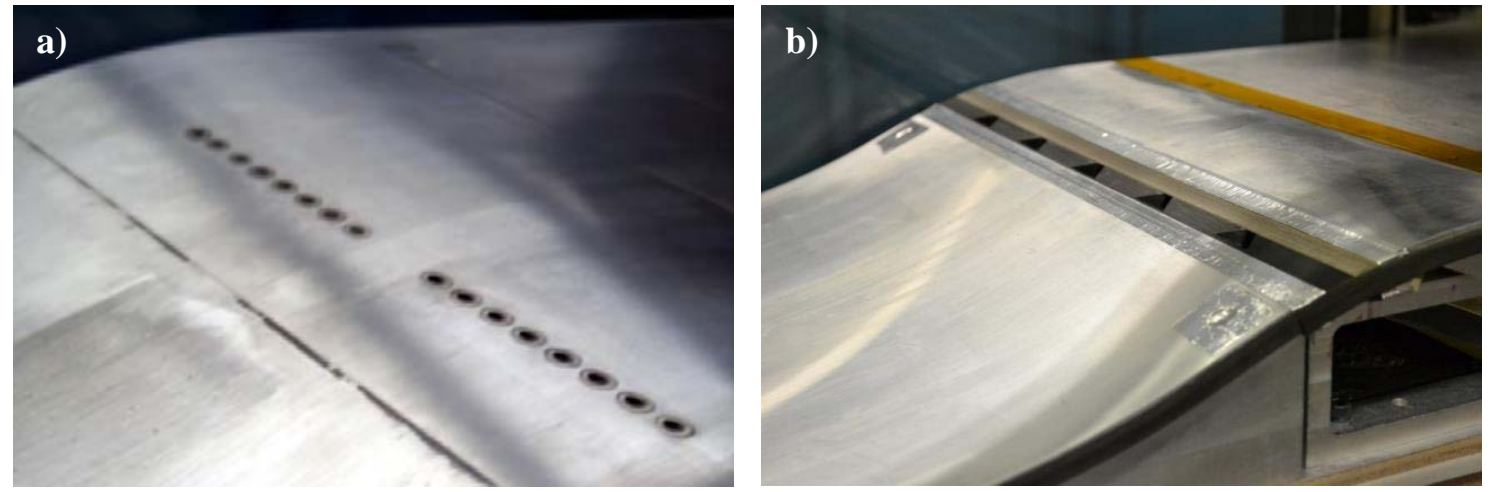

Figure 2. Adverse-pressure-gradient ramp: a) with circular orifices b) with SWJ actuator array.

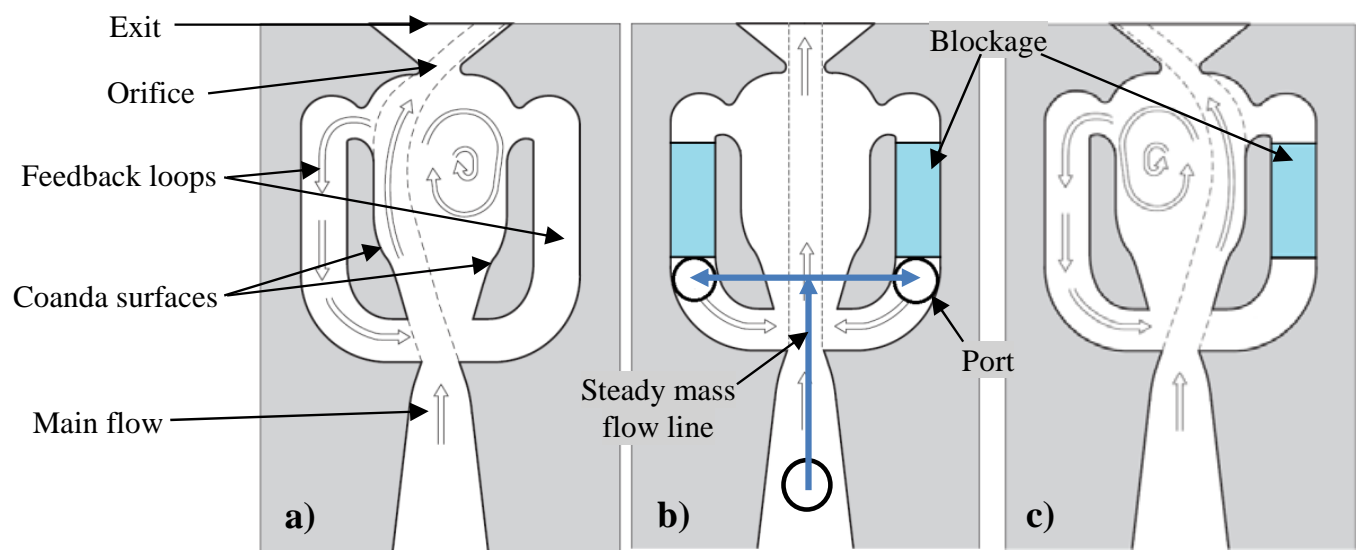

Figure 3. SWJ actuation modes: a) oscillatory blowing, b) straight blowing, c) angled blowing.

15

American Institute of Aeronautics and Astronautics 


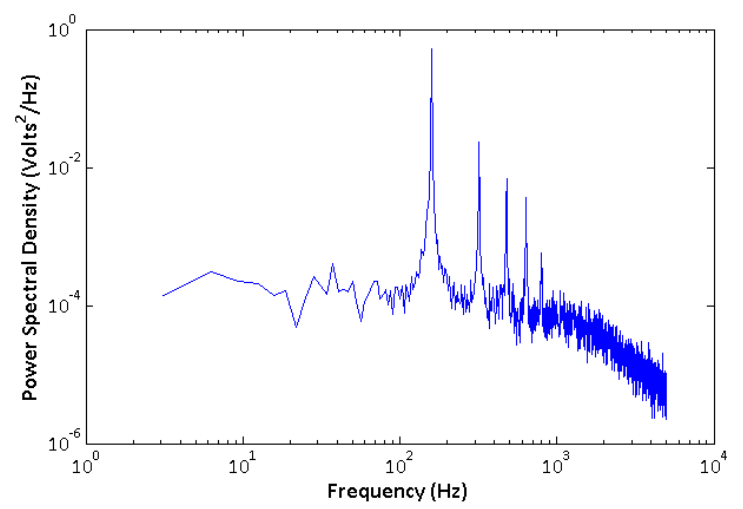

Figure 4. Power spectral density of the SWJ actuator frequency response.

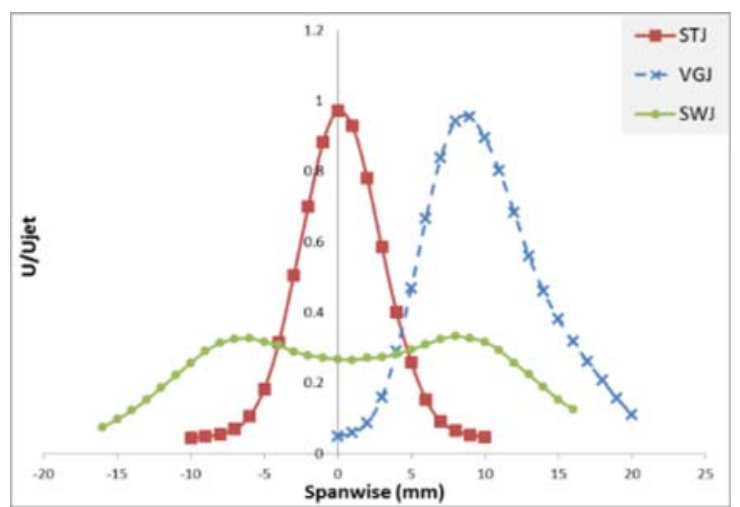

Figure 6. Normalized mean velocity profiles for three different blowing methods.

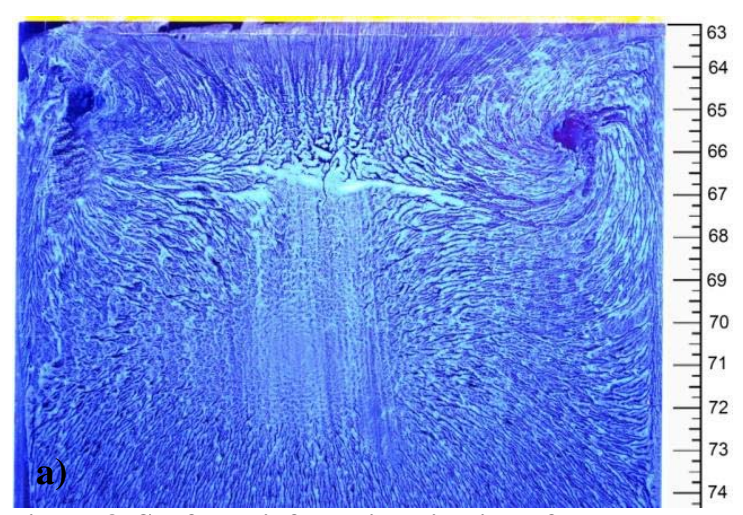

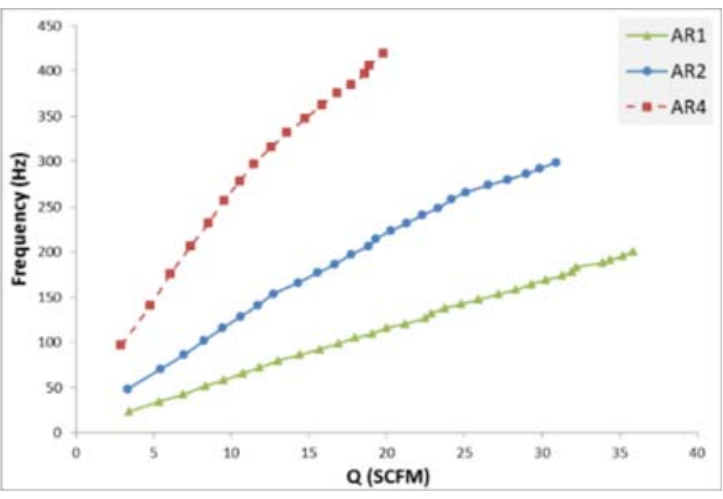

Figure 5. Variation of SWJ actuator frequency with flow rate for three different aspect ratios.

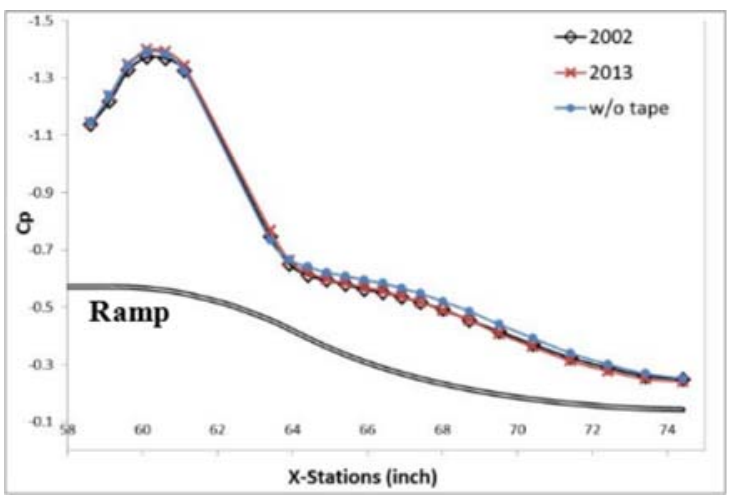

Figure 7. The comparison of the centerline $C p$ distributions for baseline cases.

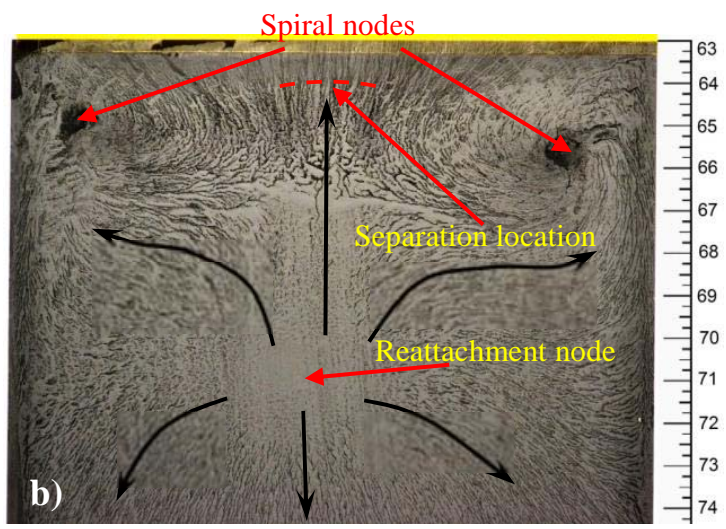

Figure 8. Surface oil flow visualization of the separated flow: a) UV light, b) white light. 


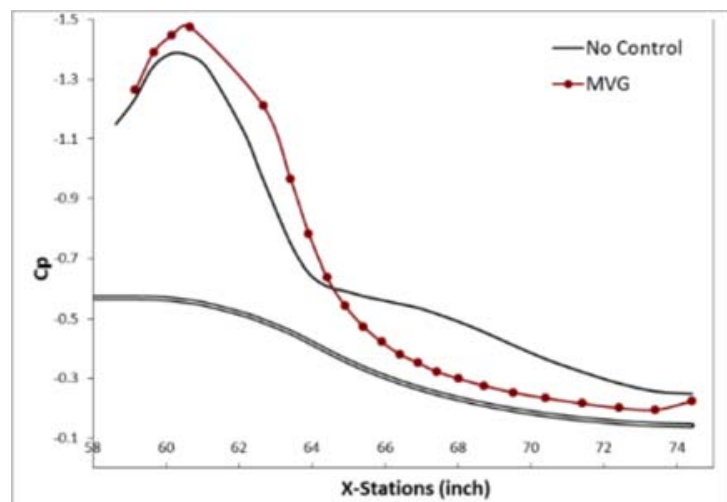

Figure 9. Centerline $\mathrm{Cp}$ distribution along the ramp for no control and MVG control cases.

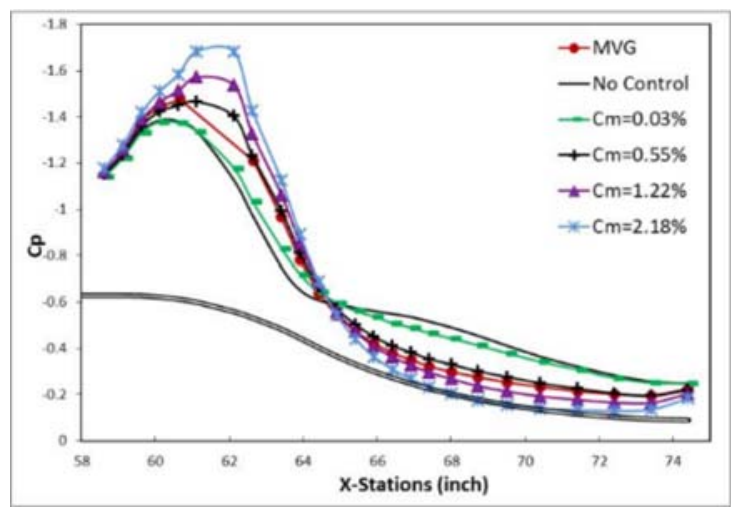

Figure 11. Effect of momentum coefficient on the Cp distribution (steady CRJ-with 8 orifices).

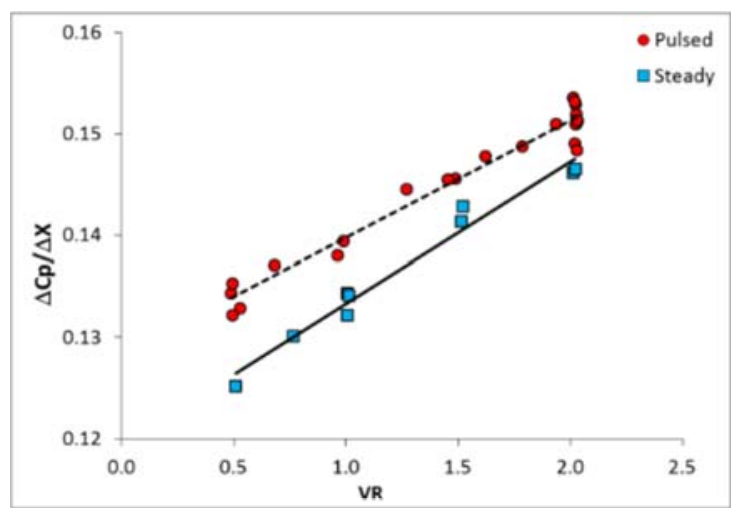

Figure 13. The comparison of steady and unsteady blowing using circular orifices $(\mathrm{Cm}=0.03$ to $0.54 \%)$.

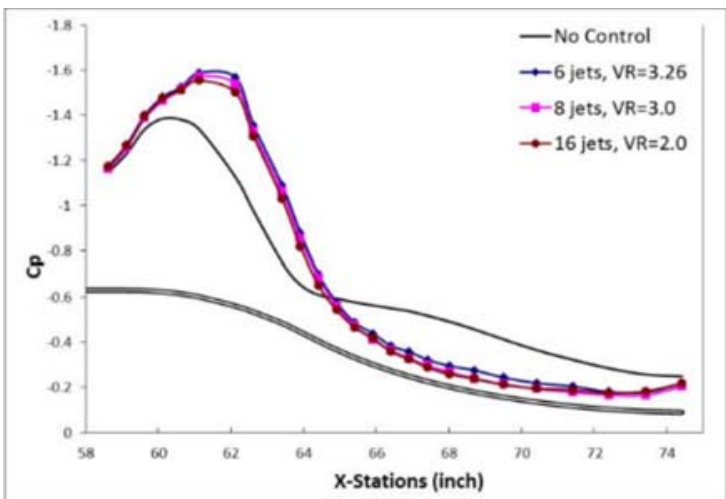

Figure 10. Effect of jet spacing on the $\mathrm{Cp}$ distribution at fixed $\mathbf{C m}=1.1 \%$.

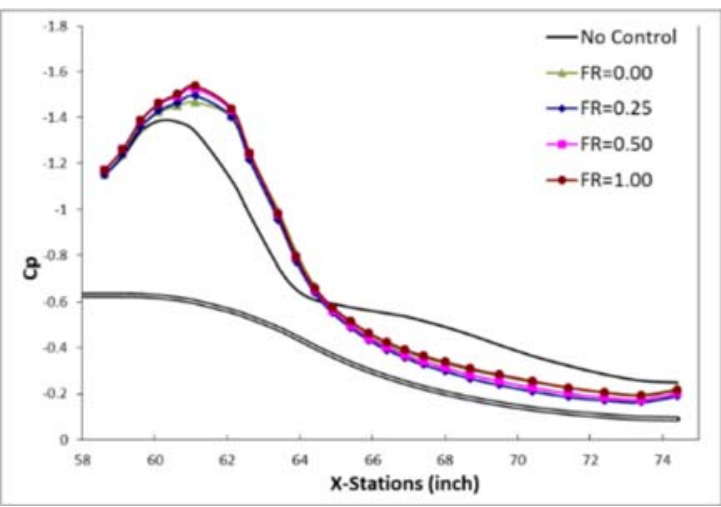

Figure 12. Effect of unsteady blowing on the Cp distribution (unsteady CRJ, $\mathrm{Cm}=0.55 \%$ ).

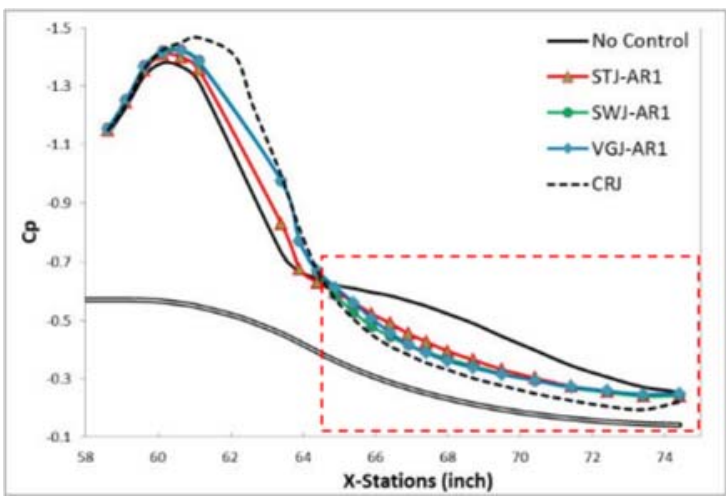

Figure 14. The comparison of steady and oscillatory blowing using SWJ-AR1 actuators, $\mathrm{Cm}=\mathbf{0 . 5 5 \%}$.

17

American Institute of Aeronautics and Astronautics 


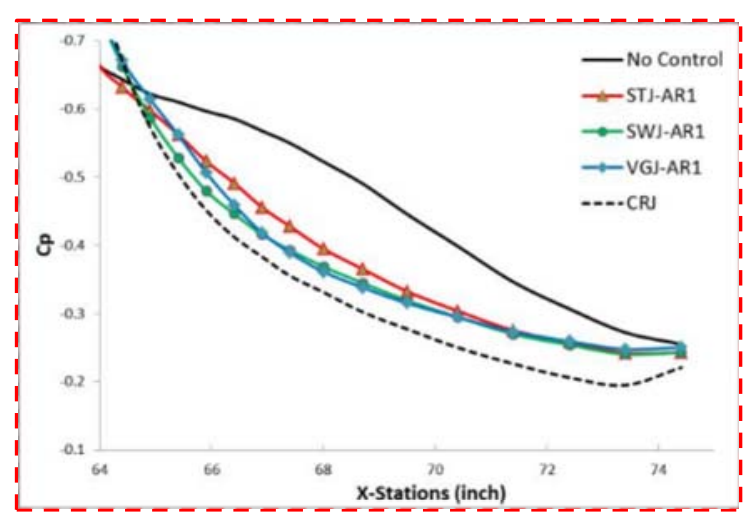

Figure 15. Close-up view of Fig.14.

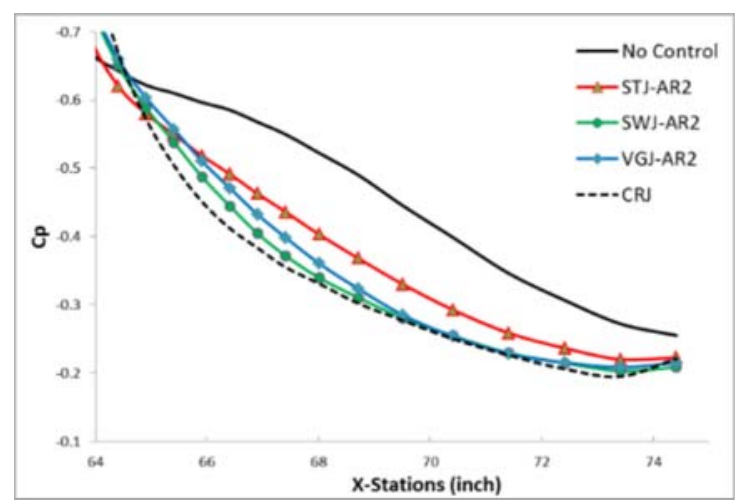

Figure 17. The comparison of steady and oscillatory blowing using SWJ-AR2 actuators, $\mathrm{Cm}=\mathbf{0 . 5 5 \%}$.

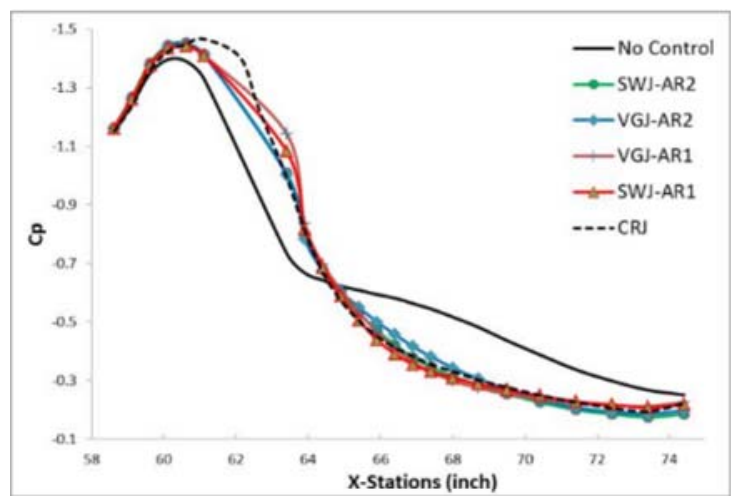

Figure 19. The comparison of the AR1 and AR2 actuators at higher $\mathrm{Cm}(1.2 \%)$.

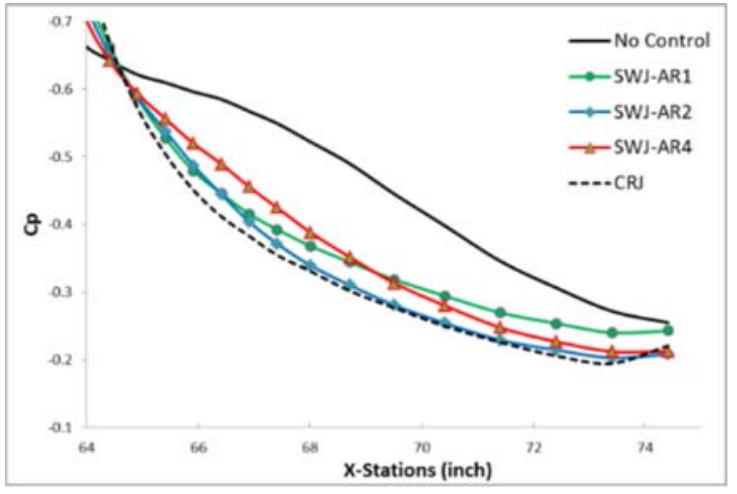

Figure 16. Variation of $\mathrm{Cp}$ distribution with aspect ratio for oscillatory blowing, $\mathrm{Cm}=\mathbf{0 . 5 5 \%}$.

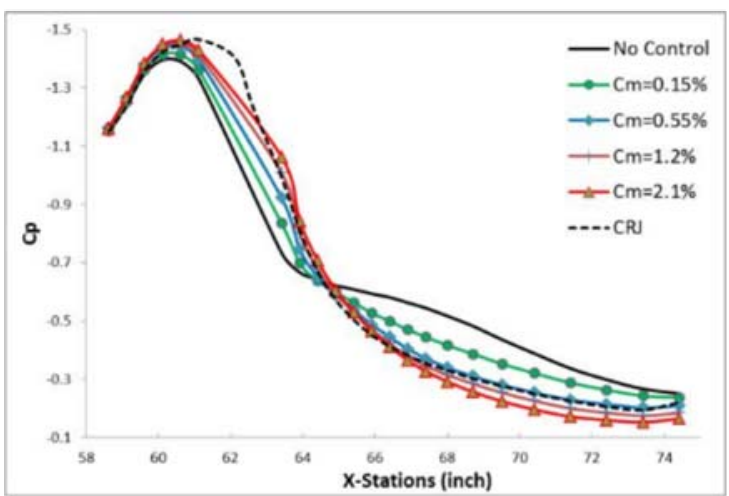

Figure 18. Effect of momentum coefficient on the $\mathbf{C p}$ distribution with SWJ-AR2 actuators.

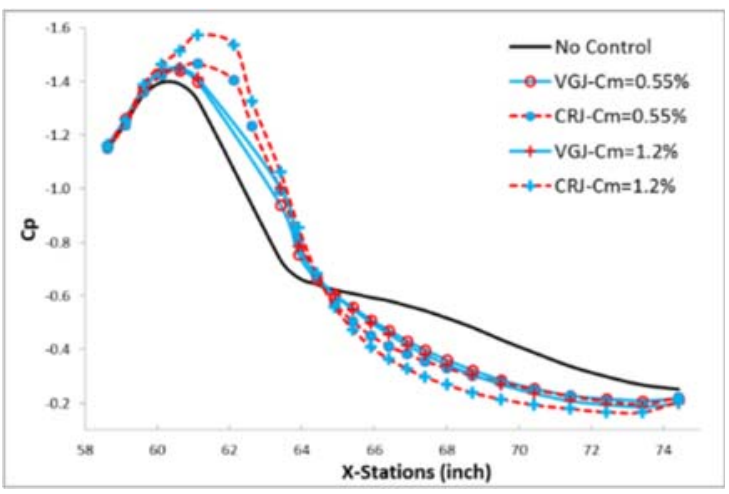

Figure 20. The comparison of two VGJ methods (Circular orifices vs. SWJ actuators).

18

American Institute of Aeronautics and Astronautics 


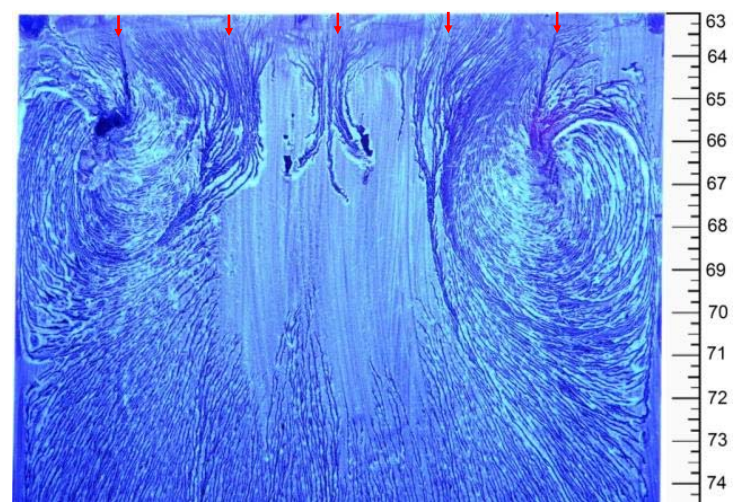

Figure 21. Oil flow visualization: Straight blowing with $\mathrm{AR}=2$ actuators, $\mathrm{Cm}=0.55 \%$.

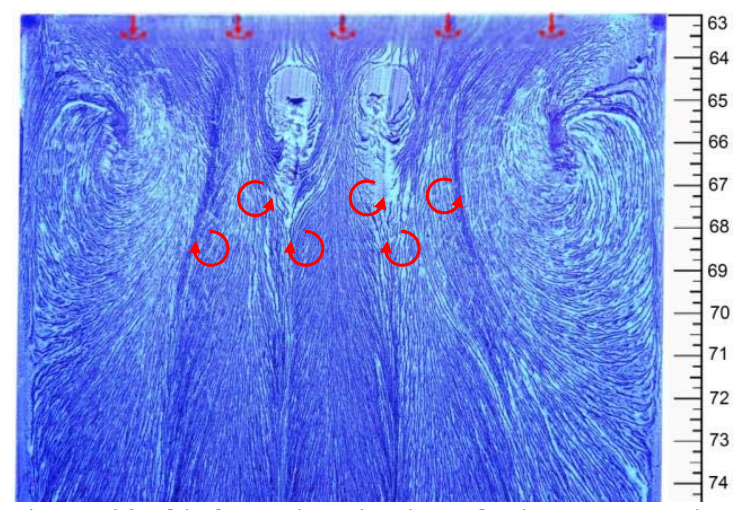

Figure 23. Oil flow visualization: Oscillatory blowing with $\mathrm{AR}=2$ actuators, $\mathrm{Cm}=\mathbf{0 . 5 5 \%}$.

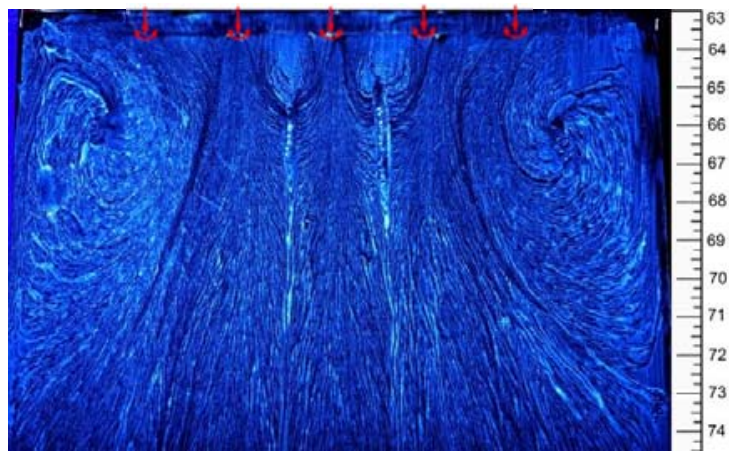

Figure 25. Oil flow visualization: Oscillatory blowing with $A R=1$ actuators, $C m=0.55 \%$.

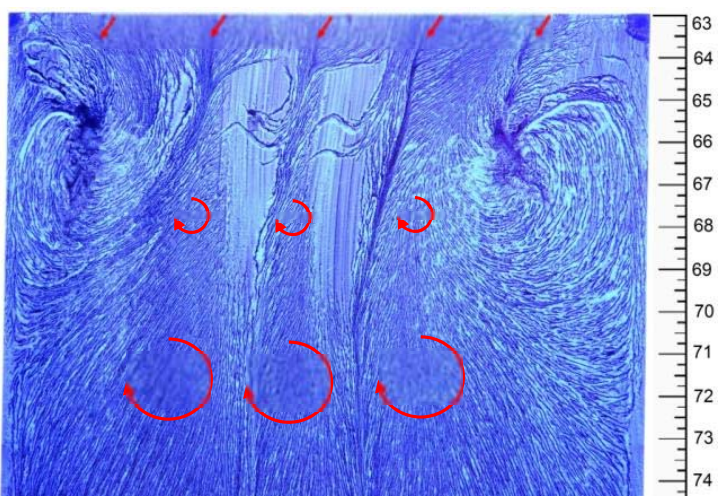

Figure 22. Oil flow visualization: Angled blowing with $\mathrm{AR}=2$ actuators, $\mathrm{Cm}=\mathbf{0 . 5 5 \%}$.

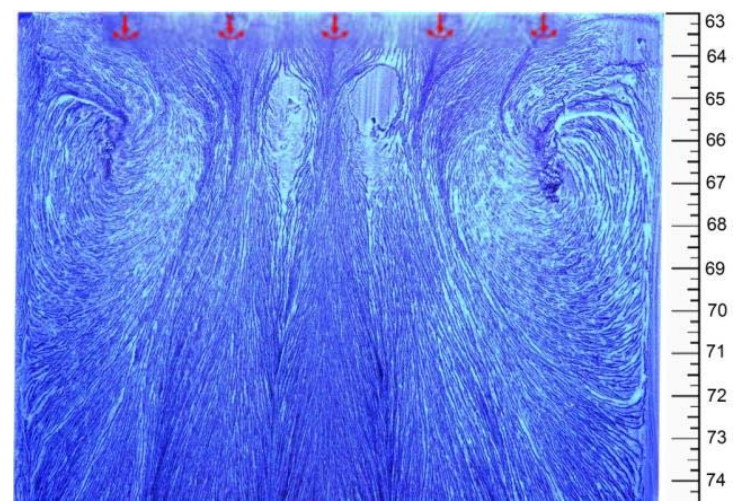

Figure 24. Oil flow visualization: Oscillatory blowing with $\mathrm{AR}=2$ actuators, $\mathrm{Cm}=1.2 \%$.

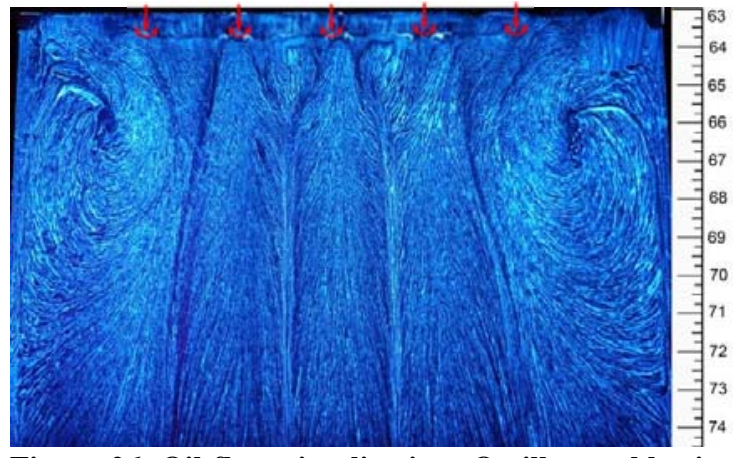

Figure 26. Oil flow visualization: Oscillatory blowing with $A R=1$ actuators, $C m=1.2 \%$. 